\title{
SEGURIDAD ALIMENTARIA Y PRODUCCIÓN AGRARIA EN EL MAGREB CENTRAL: CARACTERIZACIÓN, EVOLUCIÓN, RESTRICCIONES, POTENCIAL
}

\author{
Francisco González y Carlos Rojo ${ }^{2}$ \\ Escuela de Ingeniería Agrícola, UPM
}

\begin{abstract}
Resumen:
La seguridad alimentaria es considerada como elemento de bienestar de la población e incluso de estabilidad social y política. La agricultura tiene un papel esencial en la consecución de la seguridad alimentaria de una región o de un país; por ello, el presente trabajo analiza el sector agrario en los países del Magreb central (Argelia, Marruecos y Túnez) y su papel tanto en la seguridad como en la autosuficiencia alimentaria. En primer lugar, se revisan algunos indicadores macroeconómicos que guardan relación directa con la seguridad alimentaria y que muestran los principales aspectos estructurales del sector y el papel que desempeña la agricultura en la economía de estos tres países. A continuación se realiza un estudio de los principales productos agrícolas y ganaderos, indicando su aportación en términos de superficie, producción y rendimiento. Finalmente se analizan algunas restricciones impuestas por los condicionantes ambientales a la seguridad alimentaria y el grado de utilización de los recursos de tipo tecnológico, y se aportan algunas indicaciones de las actuaciones que se llevan a cabo y de las vías potenciales de mejora del sector agropecuario.
\end{abstract}

Palabras clave: Comercio de Alimentos, Magreb, Unión Europea, Marruecos, Argelia, Túnez, Agricultura.

Title in English: "Maghreb States Perspectives Regarding Imports and Exports of Food Products"

Abstract:

Food security is seen as an element of population welfare and even of social and political stability. Agriculture has an essential role in the aim of food security for a region or a country; in this paper the agricultural sector in the countries of Central Maghreb (Algeria, Morocco and Tunisia) and its role in their food security as well as self sufficiency is analyzed. First, some macroeconomic indicators directly related with food security, showing the main structural aspects of primary sector and the role of agriculture in the economy of the three countries are reviewed. Afterwards a study is made of the basic agricultural and livestock products, pointing their contribution in terms of surface, production and yield. Finally, some restrictions to food security based on environmental conditions and utilization level of technological resources are analyzed, providing some indications of actual and potential lines for improvement of the agrarian sector.

Keywords: Food trade, Maghreb, EU, Morocco, Algeria, Tunisia, Agriculture.

Copyright $\odot$ UNISCI, 2013.

Las opiniones expresadas en estos artículos son propias de sus autores, y no reflejan necesariamente la opinión de UNISCI. The views expressed in these articles are those of the authors, and do not necessarily reflect the views of UNISCI.

\footnotetext{
${ }^{1}$ Carlos Rojo es profesor titular del Departamento de Producción Vegetal: Fitotecnia de la Escuela de Ingeniería Técnica Agrícola de la Universidad Politécnica de Madrid y miembro del Foro Hispano-Argelino.

Dirección: Escuela de Ingeniería Técnica Agrícola UPM, Ciudad Universitaria, s/n 28040 Madrid, España E-mail: carlos.rojo@upm.es.

${ }^{2}$ Francisco González es el director de la Escuela de Ingeniería TécnicaAgrícola de la Universidad Politécnica de Madrid y miembro del Foro Hispano-Argelino.

Dirección: Escuela de Ingeniería Técnica Agrícola UPM, Ciudad Universitaria, s/n 28040 Madrid, España

E-mail: director.agricolas@upm.es.

http://dx.doi.org/10.5209/rev_UNIS.2013.n31.44700
} 


\section{Introducción}

A partir de su independencia, estos tres estados del Magreb han tenido que elaborar políticas de desarrollo conducentes a su modernización y a mejorar el nivel de vida de la población, pero no ha sido hasta la década de los años ochenta, en el siglo pasado, cuando se han llevado a cabo reformas políticas y económicas encaminadas a la liberalización de los sectores productivos. En dichas reformas se ha prestado mayor atención a la agricultura como motor del crecimiento económico y de creación de empleo y como elemento básico de seguridad alimentaria, tomando conciencia de que las sucesivas crisis regionales o globales y el incremento de los precios, -principalmente los de los granos de cereales, de los que son fuertemente dependientes-, podrían implicar serias restricciones para asegurar el suministro de alimentos a la población.

Realizar un diagnóstico sobre la situación y perspectivas del sector agrario en los países del Magreb central es complejo; dicho sector presenta ciertas similitudes en los tres países y, dada su pertenencia a la cuenca mediterránea, las condiciones bioclimáticas son parecidas a las de otros países del área, con producciones agrícolas similares: cereales, olivo, frutas, hortalizas, etc. Sin embargo, podría decirse que la diversidad de situaciones constituye el carácter dominante de la agricultura del Magreb y que dicha diversidad está originada tanto por la singularidad de los factores naturales propios como por el distinto nivel de desarrollo económico alcanzado por cada uno de países, así como por los diferentes niveles de transformación de la agricultura resultante de las planes de estímulo a la misma puestos en marcha en los últimos años. No obstante, es bien clara la necesidad de realizar dicho diagnóstico con cierta precisión, ya que la agricultura de estos tres países desempeña un papel básico ${ }^{3}$; pues se encuentra en el cruce de la mayor parte de las interacciones económicas, políticas, territoriales y culturales que afectan al área y cumple un papel estratégico en los equilibrios sociales.

Sin embargo, ese papel de equilibrio del sector agrario a veces es complejo, ya que en general se asienta sobre una agricultura con escaso grado de modernización y tecnificación, en su mayor parte extensiva, con una oferta rígida y prácticamente invariable en su composición y que se enfrenta a una demanda creciente, como resultado de las enormes presiones demográficas en la zona. Esto ha derivado, en muchos casos, a recurrir masivamente a importaciones de materias primas y alimentos que, en ocasiones, se realizan con unos costes excesivos que agravan el problema del déficit en la balanza comercial.

No obstante, la agricultura en la zona merece un tratamiento detallado por su importancia en términos de aportación al PIB y población ocupada. En Marruecos concentra cerca de la mitad de la mano de obra, pero sólo aporta un 10-15 \% del PIB, en función de las lluvias, un desequilibrio que muestra su baja productividad. En Túnez, las cifras están más equilibradas, pues ocupa un $16 \%$ de la mano de obra y un porcentaje semejante del PIB. En el resto de la región el peso de la agricultura es reducido, aunque Argelia ha acometido un ambicioso plan de desarrollo agrícola que está dinamizando el sector, en un intento de reducir su fuerte dependencia alimentaria.

En general, el Magreb cuenta con una agricultura dual, en la que conviven una agricultura moderna de exportación, dedicada a productos mediterráneos (frutas, aceite de

\footnotetext{
${ }^{3}$ Abis, Sebastien: "Un vistazo a la situación agrícola y rural mediterránea", $A F K A R / I D E A S$, Revista trimestral para el diálogo entre el Magreb, España y Europa (Invierno de 2006), pp. 76-79; Hervieu, B.; Capone, R.; Abis, S.: "Mutations et défis pour l'Agriculture au Maghreb", Les notes d'analyse du CIHEAM, no 16 (Oct. 2006).
} 
oliva, hortalizas), y una agricultura tradicional, dedicada a los cereales. Esta dualidad plantea serias limitaciones a su liberalización.

La agricultura de exportación está más capitalizada, tiene riego y condiciones climáticas favorables y encuentra sus mercados en la Unión Europea. Representa un porcentaje importante de las exportaciones de Marruecos y, en menor medida, de Túnez (15\% y $8 \%$, respectivamente, en 2005). La agricultura tradicional dedicada a los cereales obtiene bajos rendimientos, está a expensas de las frecuentes sequías, se ve sometida a tensiones contradictorias por parte de las políticas de precios de los gobiernos y resulta penalizada por las políticas de precios estatales al consumo, que mantienen artificialmente bajo el precio de los alimentos básicos como forma de redistribución de rentas a la población urbana. Junto a ellas, residualmente, persiste una agricultura de subsistencia, basada en el autoconsumo y el trueque. La conjunción de una agricultura moderna de exportación y una agricultura tradicional cerealista de bajísima productividad con los fuertes ritmos de crecimiento demográfico y urbanización ha supuesto la aparición de los déficits alimentarios. Todos los países del Magreb requieren de ingentes importaciones de alimentos para alimentar a sus poblaciones.

Ante tal problemática, las políticas agrícolas de la zona han orientado su actuación en dos vertientes: primero, estimulando la producción de aquellos productos que exigía el mercado interior, principalmente a través de subsidios, con el fin de ganar en seguridad alimentaria; segundo, acentuando al máximo la especialización en los productos de exportación en los que se disponía de alguna ventaja comparativa, lo que permitiría obtener divisas que financiasen las necesidades de importación de productos básicos, aunque en estas producciones intensivas aún conviven, junto a tecnologías avanzadas, prácticas ancestrales.

En muchos casos estas producciones intensivas han estado condicionadas también por los Acuerdos Bilaterales de Cooperación de los tres países con la Unión Europea, que la convierte, generalmente en el primer importador de frutas y hortalizas (sobre todo) procedentes de ellos. Por otra parte esto tiene cierta lógica, pues la agricultura de los países del Magreb central es típicamente mediterránea, con unas condiciones climáticas parecidas, aunque más áridas, que los países vecinos de la ribera norte, lo que conduce a que los cultivos sean similares.

En la última década se han realizado importantes iniciativas, tanto en Argelia como en Marruecos, donde a partir de 2008 se ha puesto en marcha el "Plan Maroc Vert" ${ }^{4}$. Pero a pesar de estos esfuerzos, es previsible que la rápida expansión de la demanda alimentaria y el crecimiento del consumo, resultante principalmente del crecimiento de la población y de los ingresos, y la dificultad para aumentar la oferta (por restricciones climáticas, tecnológicas, cambio climático, etc.), significará en los países del Magreb mayores dificultades para cubrir satisfactoriamente los niveles de autosuficiencia, sobre todo para los productos de base, como los cereales y el azúcar.

\subsection{Indicadores macroeconómicos ligados con la seguridad alimentaria}

Tras la aplicación de políticas conducentes a la estabilización de la economía, los países del MAGREB llevan varias décadas (especialmente desde los años 80) poniendo en marcha reformas administrativas, políticas y económicas que conduzcan a una liberalización del sistema económico y les permitan entrar en ritmos estables de crecimiento sostenido.

\footnotetext{
${ }^{4}$ Moreno Torregrosa, Pasqual (2010): "Los desafíos de la agricultura marroquí", en Desarrollo, seguridad alimentaria e internacionalización en el Magreb, Valencia, La Nau Solidària. pp. 103-124.
} 
Estas reformas, realizadas bajo los auspicios del FMI y del Banco Mundial, han tenido por objeto:

- Restaurar un crecimiento económico sostenible y reducir el desempleo,

- Recortar la inflación hasta niveles razonables,

- Mejorar la situación de la balanza de pagos y amortiguar el impacto de las reformas sobre los sectores más vulnerables de la sociedad, y

- Generar un sector privado más dinámico (especialmente en Argelia).

Esas reformas han sido evaluadas por los organismos internacionales a lo largo de los últimos años. En concreto, en Argelia en el año 2005, por el FMI (durante la presidencia de Rodrigo Rato), concluyendo que se detectaba:

- Economía más abierta y de mercado

- Avance para restaurar la estabilidad macroeconómica

- Progreso en la liberalización del comercio

- Ha crecido la economía

En los cuadros siguientes se observa que, por lo que se refiere a dos indicadores macroeconómicos básicos -tasa de crecimiento e inflación- ha habido mejoras. Se puede decir que ambos, tasa de inflación e índice de crecimiento, en esta época son bastante parecidos a los que pudiera tener un país que está en una relativa y sostenible tasa de crecimiento económico (parecido a los países de la UE en esos años, no ahora). 
Tabla 1. Indicadores macroeconómicos en Argelia durante los últimos años

\begin{tabular}{|c|c|c|c|}
\hline Año & $\begin{array}{c}\text { Tasa de inflación } \\
(\%)\end{array}$ & $\begin{array}{c}\text { Tasa de } \\
\text { crecimiento PIB } \\
(\%)\end{array}$ & $\begin{array}{c}\text { Tasa de desempleo } \\
(\%)\end{array}$ \\
\hline 2001 & 4,2 & 2,6 & 27,3 \\
\hline 2002 & 1,4 & 4,7 & 23,7 \\
\hline 2003 & 2,6 & 6,9 & 17,7 \\
\hline 2004 & 3,6 & 5,2 & 15,3 \\
\hline 2005 & 3,5 & 5,1 & 12,6 \\
\hline 2006 & 2,53 & 4,8 & 11,8 \\
\hline 2007 & 3,25 & 4,5 & 12,5 \\
\hline 2008 & 3,50 & 5,2 & 10,2 \\
\hline 2009 & 4,50 & 2,2 & 9,9 \\
\hline 2010 & 5,70 & 3,3 & \begin{tabular}{c} 
(2, \\
\hline
\end{tabular} \\
\hline
\end{tabular}

Fuente: Oficina Económica y Comercial de España en Argel 
Tabla 2. Crecimiento del PIB y evolución de la inflación en Marruecos y Túnez durante los últimos años

\begin{tabular}{|c|c|c|c|c|}
\hline \multirow[t]{2}{*}{ Año } & \multicolumn{2}{|c|}{ Marruecos } & \multicolumn{2}{|c|}{ Túnez } \\
\hline & $\begin{array}{c}\text { Crecimiento PIB } \\
(\%)\end{array}$ & Inflación (\%) & $\begin{array}{c}\text { Crecimiento PIB } \\
(\%)\end{array}$ & Inflación (\%) \\
\hline 1995 & $-6,6$ & & 2,4 & \\
\hline 1996 & 12,2 & & 7,1 & \\
\hline 1997 & $-2,2$ & & 5,4 & \\
\hline 1998 & 7,7 & & 4,8 & \\
\hline 1999 & $-0,1$ & & 6,1 & \\
\hline 2000 & 1,0 & & 4,7 & \\
\hline 2001 & 6,3 & & 4,9 & \\
\hline 2002 & 3,2 & & 1,7 & \\
\hline 2003 & 5,5 & & 5,6 & \\
\hline 2004 & 4,2 & & 6,0 & \\
\hline 2005 & 1,7 & & 4,2 & \\
\hline 2006 & 7,3 & 1,1 & 5,7 & 4,5 \\
\hline 2007 & 3,3 & 2,5 & 6,3 & 3,2 \\
\hline 2008 & 5,6 & 3,7 & 4,6 & 5,0 \\
\hline 2009 & 4,9 & 1,0 & 3,0 & 3,7 \\
\hline 2010 & 3,3 & 2,2 & & \\
\hline 2011 & 4,8 & 0,9 & & \\
\hline
\end{tabular}

Fuente: Oficina Económica y Comercial de España en Rabat ${ }^{5}$, Oficina Económica y Comercial de España en Túnez

\footnotetext{
${ }^{5}$ Secretaría de Estado de Comercio: "Guía País Marruecos", Oficina Económica y Comercial de España en Rabat (Junio 2012).
} 
En Marruecos, la dependencia del PIB de la agricultura es muy superior a la de otros dos países, especialmente Argelia, que depende del petróleo y gas. Por tanto, en Marruecos existen mayores fluctuaciones en el PIB, asociadas a condiciones climáticas especialmente adversas para la agricultura, como se observa en los años 1995, 2001 y 2005, en los que se produjeron episodios de sequía.

Sin embargo, las reformas a las que se ha hecho referencia al inicio de este apartado no tuvieron un impacto positivo sobre la situación del desempleo hasta casi una década después de su implantación, pues hasta la mitad de la década pasada (2004-2005) se mantuvieron en cifras comprendidas entre 25-30 \%, en Argelia, (Tabla 1) y en torno al 20\% en Marruecos; a partir de estos años es cuando descendieron de forma significativa a los valores actuales, en torno al $10 \%$ en Argelia, el $9 \%$ en Marruecos y el $14 \%$ en Túnez, donde curiosamente se ha pasado de una tasa ligeramente superior al $3 \%$ en el año 2000 a esta otra; es muy posible que en Túnez los datos sean más fiables, ya que aunque, como se ve, el desempleo ha ido reduciéndose hasta situarse en las tasas citadas, se trata de cifras muy contestadas, especialmente en Marruecos y Argelia. Se estima que incluyen muchas situaciones, especialmente relacionadas con trabajos a domicilio y actividades en el medio rural, que no son registradas como desempleo, por lo que se considera que las tasas reales podrían acercarse al $25-30 \%$.

Uno de los principales problemas actuales es precisamente el desempleo, que alcanza niveles muy elevados y se concentra en los jóvenes y las mujeres. En el medio rural los datos están mediatizados por la falta de estadísticas, el desempleo encubierto, el subempleo y las actividades de subsistencia. El mercado de trabajo es incapaz de absorber la gran cantidad de jóvenes que se incorporan al mismo como fruto del dinamismo demográfico. Esta situación genera tensiones sociales y supone un factor de expulsión que desemboca en corrientes migratorias.

Hasta la fecha, el crecimiento del PIB ha sido insuficiente para generar los recursos exigidos por la presión demográfica. En el largo plazo, las economías del Magreb han crecido por debajo del ritmo de otros países en desarrollo. A título de ejemplo, Marruecos y Corea del Sur partían de niveles de PIB per cápita similares en 1960. Con datos del PNUD para el período 1975-2004, la tasa de crecimiento anual medio del PIB per cápita fue del $6 \%$ en Corea del Sur, por sólo el 0,1 \% en Argelia, el 1,4 \% en Marruecos y el 2,3 \% en Túnez.

La constatación de todo ello es que el Magreb presenta índices de desarrollo humano bajos, que se explican sobre todo por la incidencia del analfabetismo, que afecta principalmente a la población rural y a las mujeres. De un total de 177 países recogidos en el Índice de Desarrollo Humano (IDH) del PNUD en 2005, Argelia ocupaba el puesto 102, Marruecos el 123 y Túnez el 87. En ese año, la renta per cápita en paridad de poder adquisitivo era en Argelia de 6.603 dólares, 4.309 en Marruecos y 7.768 en Túnez. Pese a que los países del Magreb destinan una parte importante de su PIB al gasto en educación, la tasa de alfabetización entre adultos apenas supera el 70\% en Argelia, el 82\% en Libia, el 52\% en Marruecos y el $74 \%$ en Túnez.

\subsection{El sector agrario}

La importancia relativa de este sector, comparada con el resto de países del área mediterránea, se observa en la Tabla 3.

\footnotetext{
${ }^{6}$ Secretaría de Estado de Comercio: "Guía País Túnez", Oficina Económica y Comercial de España en Túnez (Septiembre 2012).
} 
Tabla 3. Comparación de los diferentes parámetros macroeconómicos en los países del área mediterránea

\begin{tabular}{|c|c|c|c|c|c|c|c|c|}
\hline País & $\begin{array}{c}\text { Tasa de } \\
\text { crecimiento } \\
\text { del PIB (\%) }\end{array}$ & $\begin{array}{c}\text { PIB } \\
\text { agricola } \\
\text { total } \\
(\%)\end{array}$ & $\begin{array}{c}\text { Crecimiento } \\
\text { de la } \\
\text { población } \\
(\%)\end{array}$ & $\begin{array}{c}\text { Población } \\
\text { agraria } \\
\text { /Total }(\%)\end{array}$ & $\begin{array}{c}\text { Tasa de } \\
\text { desempleo } \\
(\%)\end{array}$ & $\begin{array}{c}\text { Inflación } \\
(\%)\end{array}$ & $\begin{array}{c}\text { PIB } \\
\text { /Activos }\end{array}$ & $\begin{array}{c}\text { PIB } \\
\text { Activos } \\
\text { agrarios }\end{array}$ \\
\hline Albania & 4,1 & 54,0 & 0,2 & 68,0 & n.d. & n.d. & 1.973 & 2.176 \\
\hline Argelia & 3,8 & 9,3 & 1,8 & 21,0 & 30,0 & 0,4 & 4.676 & 2.341 \\
\hline Egipto & 3,9 & 17,0 & 1,2 & 37,0 & n.d. & n.d. & 3.536 & 1.768 \\
\hline España & 4,1 & 3,3 & 0,1 & 7,1 & 14,1 & 3,6 & 34.429 & 17.365 \\
\hline Francia & 3,1 & 2,2 & 0,4 & 3,4 & 8,8 & 1,5 & 53.963 & 35.595 \\
\hline Grecia & 4,1 & 7,2 & 0,2 & 17,0 & 11,0 & 2,9 & 27.173 & 11.264 \\
\hline Italia & 2,9 & 2,4 & 0,0 & 5,7 & 10,5 & 2,5 & 46.524 & 21.730 \\
\hline Líbano & 0,0 & 12,0 & 1,7 & 9,0 & 0,3 & 0,3 & 14.145 & n.d. \\
\hline Malta* & 4,0 & n.d. & 2,6 & 6,7 & n.d. & n.d. & 24.803 & n.d. \\
\hline Marruecos & 0,8 & 10,8 & 1,8 & 37,7 & 21,5 & 1,9 & 3.045 & 4.073 \\
\hline Portugal & 3,3 & 2,8 & 0,2 & 10,0 & 4,0 & 2,9 & 22.484 & 5.406 \\
\hline Túnez & 5,9 & 12,8 & 1,2 & 20,0 & 3,3 & 3,3 & 5.612 & 2.693 \\
\hline & 7,0 & 13,5 & 1,5 & 35,8 & 6,5 & 23,7 & 6.492 & 1.805 \\
\hline
\end{tabular}

Fuente: Elaborado por Malorgio (2002) ${ }^{7}$. Datos de 1999

De esta Tabla se deduce, en primer lugar, que la aportación de la agricultura al PIB en los tres países considerados es similar a la de otros países sureños, pero en proporción muy superior a la de los países de la ribera norte del Mediterráneos, excepto Albania. Por otro lado, el ritmo de crecimiento de la población es muy superior al de los países del norte. Además, el porcentaje de población agraria es todavía superior al de los países del norte, aunque sea inferior al valor en Egipto y Turquía.

\footnotetext{
${ }^{7}$ Malorgio, Giuliano (2002): "Integración y competitividad de los sistemas agroalimentarios mediterráneos", en La Agricultura Mediterránea en el Siglo XXI, Colección Mediterráneo Económico, $\mathrm{n}^{\circ}$ 2, Caja Rural Intermediterránea (CAJAMAR), pp. 13-32.
} 
De la tasas de desempleo e inflación ya se ha hecho algún comentario. En cuanto a La renta per capita, se observa que es claramente inferior a de los países de la ribera norte, pero igual que en ellos es superior en el medio urbano que el rural, excepto en Marruecos.

Otro elemento de referencia es el que surge de la comparación de los Usos del Suelo en los países del área mediterránea, cuyos datos se muestran en la Tabla 4.

Tabla 4. Comparación del uso de la tierra en los países del área mediterránea. Datos en miles de ha.

\begin{tabular}{|c|c|c|c|c|c|}
\hline País & $\begin{array}{l}\text { Superficie } \\
\text { agraria }\end{array}$ & $\begin{array}{l}\text { Superficie } \\
\text { cultivable }\end{array}$ & $\begin{array}{c}\text { Cultivos } \\
\text { permanentes }\end{array}$ & $\begin{array}{c}\text { Pastos } \\
\text { permanentes }\end{array}$ & Bosques \\
\hline Albania & 1.128 & 577 & 122 & 429 & 1.046 \\
\hline Argelia & 42.415 & 7.700 & 515 & 34.500 & 4.050 \\
\hline Egipto & 3.690 & 2.834 & 466 & n.d. & 31 \\
\hline España & 27.030 & 13.680 & 4.850 & 8.500 & 15.858 \\
\hline Francia & 29.900 & 18.361 & 1.154 & 10.385 & 14.850 \\
\hline Grecia & 9.020 & 2.762 & 1.108 & 5.150 & 2.620 \\
\hline Italia & 16.268 & 8.545 & 2.877 & 4.846 & 6.764 \\
\hline Líbano & 324 & 180 & 128 & 16 & 80 \\
\hline Malta & 11 & 10 & 1 & n.d. & 14 \\
\hline Marruecos & 30.445 & 8.500 & 945 & 21.000 & 8.000 \\
\hline Portugal & 4.142 & 1.968 & 737 & 1.437 & 3.250 \\
\hline Túnez & 9.000 & 2.850 & 2.250 & 3.900 & 668 \\
\hline Turquía & 39.050 & 24.138 & 2.534 & 12.378 & 20.199 \\
\hline
\end{tabular}

Fuente: Elaborado por Malorgio (2002) ${ }^{8}$. Datos de 1999

${ }^{8}$ Ibid. 
De la Tabla se deduce la presencia de grandes superficies agrarias, pero una reducida superficie cultivable, ya que en el total se incluye una gran superficie de pastos de carácter muy extensivo y que soportan cargas ganaderas inferiores a 1 UGM por hectárea. Esta distribución de tierras, especialmente esa gran cantidad de tierras destinadas a pastos, muy poco productivas, impone una fuerte restricción a la producción de alimentos en detrimento de la seguridad alimentaria.

Por otra parte, hay que considerar que en la superficie cultivable la estructura de las explotaciones no es la adecuada, pues aunque las cifras indican una superficie tan grande y con un grado de extensión muy acusado se observa que -por ejemplo- en Argelia, más del 70 $\%$ de las explotaciones son menores de 10 ha.

Hay que considerar asimismo la organización territorial en la zona. En el Magreb Central, el clima y los factores orográficos desempeñan un papel fundamental en la localización de las áreas en las que la agricultura tiene mejor porvenir. En las cercanías a las áreas costeras se agrupan las tierras más fértiles y los principales recursos hídricos, que se defienden del avance del desierto por las cadenas montañosas que se encuentran más al sur de esta área en cada uno de los países.

La actividad agrícola se desarrolla en su mayor parte en esas franjas costeras, donde las tierras son fértiles y las precipitaciones, aunque irregulares, permiten el desarrollo de la agricultura mediterránea. En consecuencia, la población tiende a situarse en el litoral, dejando despobladas las regiones interiores y montañosas. El período colonial exacerbó este desequilibrio territorial, al localizar las industrias en las zonas urbanas costeras dotadas de infraestructuras de transporte. Como consecuencia, el Magreb padece un doble desequilibrio territorial: entre el litoral y el interior, y entre el campo y la ciudad. Las malas condiciones de vida en el medio rural han forzado el éxodo hacia las urbes, cuyos servicios se han visto desbordados por el aluvión de emigrantes rurales y el dinamismo demográfico.

\section{La Seguridad alimentaria}

Dentro de la definición del concepto de seguridad alimentaria, la más antigua se remonta a la Declaración Universal de los Derechos Humanos del año 1948, que indicaba, en su artículo 25:

"Toda persona tiene derecho a un nivel de vida adecuado que le asegure, así como a su familia, la salud y el bienestar, y en especial la alimentación".

Posteriormente, en la Cumbre Mundial de la Alimentación del año 1974, la seguridad alimentaria fue definida desde la perspectiva del suministro de alimentos:

"Que haya en todo tiempo a nivel mundial existencias suficientes de alimentos para mantener una expansión constante del consumo y contrarrestar las fluctuaciones de la producción y los precios". 
El objetivo prioritario de esta definición es realizar una contribución a la estabilidad y disponibilidad nacional e internacional de los precios de los alimentos básicos.

Entre las definiciones más destacadas se encuentra la del Banco Mundial (1986), que interpreta la seguridad alimentaria como el acceso de todas las personas en todo momento a suficiente alimento para llevar una vida activa y sana.

En 1996, en la Cumbre Mundial de la Alimentación, la FAO definió la Seguridad Alimentaria del siguiente modo:

"Cuando todas las personas tienen en todo momento acceso físico, social y económico a los alimentos suficientes, sanos y nutritivos que satisfagan sus necesidades energéticas diarias y preferencias alimentarias para llevar una vida sana y activa".

Esta definición fundamenta la seguridad alimentaria como un derecho. Con algún pequeño matiz, la definición ha sido adoptada por la Agencia de los Estados Unidos para el Desarrollo Internacional (USAID), en 1999 y ha sido incorporada por más de 40 países en sus propios textos constitucionales; la FAO espera que sea incorporada por otros muchos.

El derecho a la alimentación consagrada en la definición de FAO de 1996, se fundamenta en cuatro pilares:

- Disponibilidad. Se refiere a la cantidad y variedad de alimentos y la estabilidad de los mismos en el tiempo y en el espacio. Este es, por tanto, un concepto que hace referencia a la oferta de alimentos, la cual viene motivada por factores como la producción, las importaciones y la ayuda alimentaria (países en desarrollo).

- Acceso. Es la capacidad de los hogares, sea por medio de sus posibilidades para producirlos, comprarlos o de acceder a ellos mediante transferencias o donaciones.

- Consumo. Está íntimamente relacionado con las costumbres, creencias, conocimientos, preferencias culturales, prácticas de alimentación y la educación de los hogares.

- Utilización biológica. hace referencia al aprovechamiento óptimo de los alimentos a nivel nutricional, el cual depende de condiciones como la salubridad del entorno (por ejemplo: el acceso al agua potable, la contaminación del entorno) y la condición de salud del individuo para aprovechar los alimentos.

A nivel internacional, en cuanto a la utilización del término seguridad alimentaria, se identifican dos acepciones diferentes en inglés, y dos dimensiones de un mismo concepto en castellano (seguridad alimentaria). La Organización Mundial de la Salud (OMS), al mencionar el término "food safety" se refería a la inocuidad de los alimentos, a la garantía de su salubridad para el consumidor; mientras que la expresión "food security", en el sentido indicado por FAO, se refiere a la disponibilidad suficiente de alimentos.

En el ámbito de la UE, el Reglamento CE 178/2002 define los diferentes elementos que constituyen la seguridad alimentaria para el conjunto de países de la UE; en él se prescinde 
del término amplio (food security) adoptando un acuerdo de un enfoque más restringido (food safety), cuya terminología abarca, entre otros elementos, la salubridad e higiene de los productos alimentarios, la alimentación y salud animal, el bienestar de los animales y la protección fitosanitaria y sanitaria a lo largo de toda la cadena alimentaria.

A simple vista, ¿cuál es la situación del Magreb central en el aspecto de la seguridad alimentaria? Podría decirse que hay suministro de alimentos, es decir, hay food security, pero quizá menos food safety, al menos en las zonas rurales (no así en las áreas urbanas).

\section{Caracterización productiva del Sector Agrario en el Magreb Central}

\subsection{Marco genérico}

Como primer elemento de referencia, hay que señalar la superficie geográfica y agrícola de cada país. Estos datos, junto con la superficie que se recolecta, figuran en la Tabla 5, con cifras más actuales que las de la Tabla 4. Se han incluido los datos de España como contraste y referencia. La superficie agrícola no recolectada se refiere a cultivos no contabilizados por FAO, pero sobre todo a áreas en barbecho y pastizales y otros aprovechamientos agrarios que no se cosechan.

Tabla 5. Características geográficas destacadas de los países del Magreb Central y de España

\begin{tabular}{|l|c|c|c|}
\hline \multicolumn{1}{|c|}{ País } & $\begin{array}{c}\text { Superficie } \\
\text { geográfica }\left(\mathrm{km}^{2}\right)\end{array}$ & $\begin{array}{c}\text { Superficie agrícola } \\
\text { (miles de ha)* }\end{array}$ & $\begin{array}{c}\text { Superficie recolectada } \\
\text { (miles de ha)** }\end{array}$ \\
\hline Argelia & 2.381 .740 & 8.435 & $4.363,1$ \\
Marruecos & 446.550 & 9.055 & $7.042,8$ \\
Túnez & 163.610 & 4.936 & $3.080,5$ \\
\hline España & 505.370 & 17.216 & $11.878,1$ \\
\hline
\end{tabular}

* Conjunto de tierras cultivables y cultivos y aprovechamientos permanentes. AQUASTAT, FAO; datos 2009. ${ }^{9}$

** Suma de superficies recolectadas de los cultivos contabilizados. FAOSTAT, FAO; datos $2010 .{ }^{10}$

\footnotetext{
${ }^{9}$ FAO (2012): Base de Datos AQUASTAT, Hojas de datos nacionales: http://www.fao.org/nr/water/aquastat/data/factsheets/.

${ }^{10}$ FAO (2012): Base de Datos FAOSTAT, Estadísticas de cultivos:

http://faostat.fao.org/site/567/default.aspx\#ancor.
} 
En el caso de Argelia (que es el país de África más extenso) se aprecia la importancia indiscutible de la superficie de desierto. En lo relativo a superficie agrícola, ninguno de los tres países magrebíes alcanza los 10 millones de ha; Túnez, que es el que tiene menor superficie total, presenta un mayor porcentaje de superficie dedicada a la agricultura (un $30 \%$ del total, proporción similar a la de España).

Otro aspecto a considerar para la producción agraria es la proporción de superficie que está en régimen de regadío. Los datos correspondientes se recogen en la Tabla 6.

Tabla 6. Superficie equipada para el riego en los países del Magreb Central, y en España

\begin{tabular}{|l|c|}
\hline \multicolumn{1}{|c|}{ País } & Superficie equipada para el riego (miles de ha) \\
\hline Argelia & 569,4 \\
Marruecos & $1.485,0$ \\
Túnez & 394,0 \\
\hline España & $3.818,0$ \\
\hline
\end{tabular}

Datos AQUASTAT, FAO. Argelia y Túnez, datos de 2001; Marruecos, datos de 2004; España, datos de 2008. ${ }^{1}$

Un factor adicional, que afecta especialmente al cultivo en secano, es el régimen pluviométrico. En la Figura 1 se muestra, de forma bastante esquemática, la pluviometría media en la zona geográfica objeto de análisis. La superficie no mostrada de Argelia tiene, evidentemente, un régimen de lluvias muy reducido.

\footnotetext{
${ }^{11}$ FAO (2012): Base de Datos AQUASTAT, Hojas de datos nacionales.
} 


\section{Figura 1. Distribución de la pluviometría media anual en el Magreb Central.}

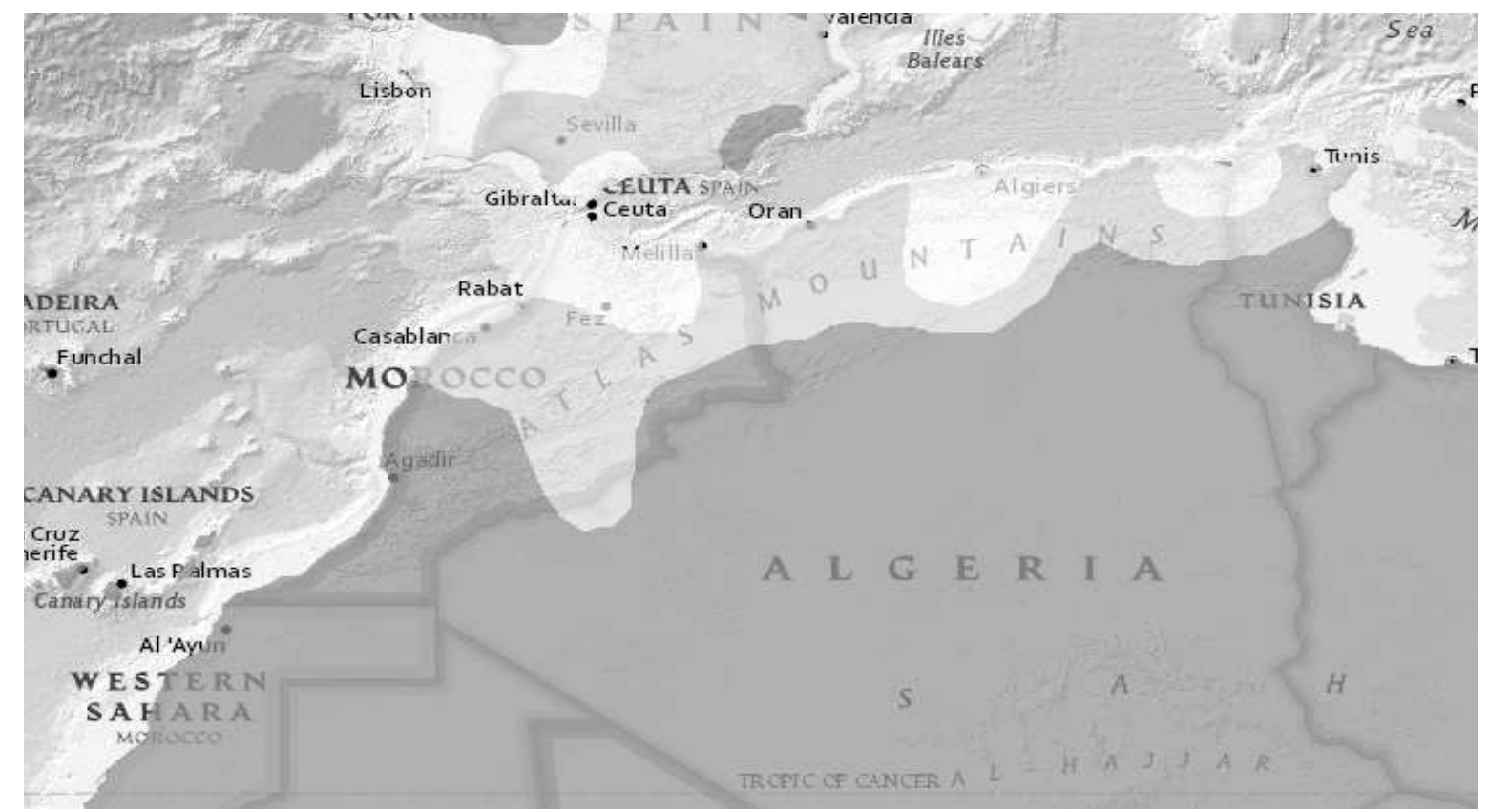

Base cartográfica: National Geographic Society. Información, United Nations Environment Program ${ }^{12}$.

Se puede apreciar que hay áreas (las más claras) en las que la pluviometría es relativamente abundante; ello ocurre en la parte de Marruecos más próxima al Estrecho de Gibraltar, hasta la cordillera del Atlas, en la zona de Argelia que rodea Argel, abarcando los Haut Plateaux hasta el Tell sahariano más cercanos y, y también en la zona montañosa próxima a la costa en el área fronteriza entre Argelia y Túnez. En estas tres áreas, las más claras en la imagen, se superan los $600 \mathrm{~mm}$ anuales de media, y en las zonas más altas del Atlas marroquí se llega a más de $1.000 \mathrm{~mm}$, con nevadas invernales.

Las pluviometrías inferiores, entre 400 y $600 \mathrm{~mm}$, circundan las zonas antes citadas, e incluyen buena parte de Marruecos, casi todo el resto de la parte de Argelia al Norte del Tell sahariano y aproximadamente el resto del tercio norte de Túnez. En la Figura 1 se corresponde con las áreas de color gris claro. El resto de los tres países tiene un régimen de lluvias inferior a los $400 \mathrm{~mm}$ anuales; con más de $200 \mathrm{~mm}$ se configura una franja de anchura variable, que circunda las zonas de más lluvia, y el resto del territorio se caracteriza por una aridez muy elevada, que se acentúa hacia el sur.

Evidentemente, el régimen de lluvias es un condicionante de primer orden de la actividad agraria. Por una parte, es un limitador directo del potencial productivo en las zonas donde no se dispone de otro suministro alternativo de agua para la vegetación, de modo que en condiciones de secano, pluviometrías de menos de 400-500 $\mathrm{mm}$ anuales no permiten un desarrollo satisfactorio de muchas especies cultivadas. Por otro lado, también supone una limitación para el potencial de regadío, en la medida en que el riego es una redistribución, en el espacio y/o en el tiempo, del agua disponible. Por último, aunque no con menos importancia, hay que considerar la disponibilidad de agua como un elemento esencial para la formación del suelo sobre el que se desarrolla la vegetación. La generación del suelo se fundamenta en la actividad biológica que transforma los materiales puramente minerales

\footnotetext{
${ }^{12}$ National Geographic Map Maker, en http://educationlnatinalgeographic.com/education/mapping/.
} 
(regolita); en la medida en que escasea la disponibilidad de agua, se reduce la actividad biológica y la velocidad de formación de suelo se ralentiza e incluso se anula.

En consecuencia, esta diferencia en la disponiblidad de agua se traduce en la ubicación histórica de las superficies de cultivo. Hay una clara correspondencia entre la disponibilidad de agua de lluvia y las áreas de desarrollo de los cultivos en los países analizados.

\subsection{Producción agrícola}

Los cultivos más importantes, por superficie ocupada, en cada uno de los tres países de la zona objeto de estudio se muestran en la Tabla 7. En los tres países, los dos cereales básicos (trigo y cebada) están en los primeros lugares. El tercer gran cultivo es el olivar, que ocupa el primer lugar, con diferencia, en el caso de Túnez. Hay que decir que tradicionalmente Túnez ha sido uno de los grandes productores mundiales de aceite de oliva.

Tabla 7. Cultivos con más superficie dedicada en los tres países del Magreb Central

\begin{tabular}{|l|r|l|r|l|r|}
\hline \multicolumn{2}{|c|}{ Argelia } & \multicolumn{2}{c|}{ Marruecos } & \multicolumn{2}{c|}{ Túnez } \\
\hline \multicolumn{1}{|c|}{ Cultivo } & Superficie & \multicolumn{1}{|c|}{ Cultivo } & Superficie & Cultivo & Superficie \\
\hline Trigo & $1.660,4$ & Trigo & $2.888,8$ & Olivo & $1.974,2$ \\
Cebada & 859,1 & Cebada & $2.107,6$ & Trigo & 720,5 \\
Olivo & 282,5 & Olivo & 600,4 & Cebada & 387,1 \\
Palmera dat. & 159,2 & Maíz & 231,9 & Almendro & 180,3 \\
Patata & 100,8 & Habas secas & 175,7 & Habas secas & 54,1 \\
Viñedo & 72,9 & Almendro & 136,0 & Pistacho & 44,2 \\
Avena & 71,0 & Garbanzos & 75,6 & Palmera dat. & 40,8 \\
\hline
\end{tabular}

Datos de superficie en miles de ha: Valores medios del período 2005-2010.

Fuente: FAOSTAT, FAO. ${ }^{13}$

Estos cultivos principales pueden servir para dar una imagen básica de las circunstancias y características de la agricultura de cada país. Los aspectos más destacados de estas especies se exponen seguidamente. En todos los casos, los datos proceden de la base de datos estadística de FAO, FAOSTAT, cuya referencia ya se ha dado.

En lo que se refiere al trigo, el Gráfico 1 muestra la evolución de superficie cultivada y producción obtenida en los tres países a lo largo de los últimos años.

\footnotetext{
${ }^{13}$ FAO (2012): Base de Datos FAOSTAT, Estadísticas de cultivos.
} 
Gráfico 1. Evolución de la superficie y producción del cultivo de trigo en Argelia $(\diamond)$, Marruecos ( $\square)$ y Túnez ( $($ ) durante los últimos años. Superficie, línea continua; producción, línea de trazos

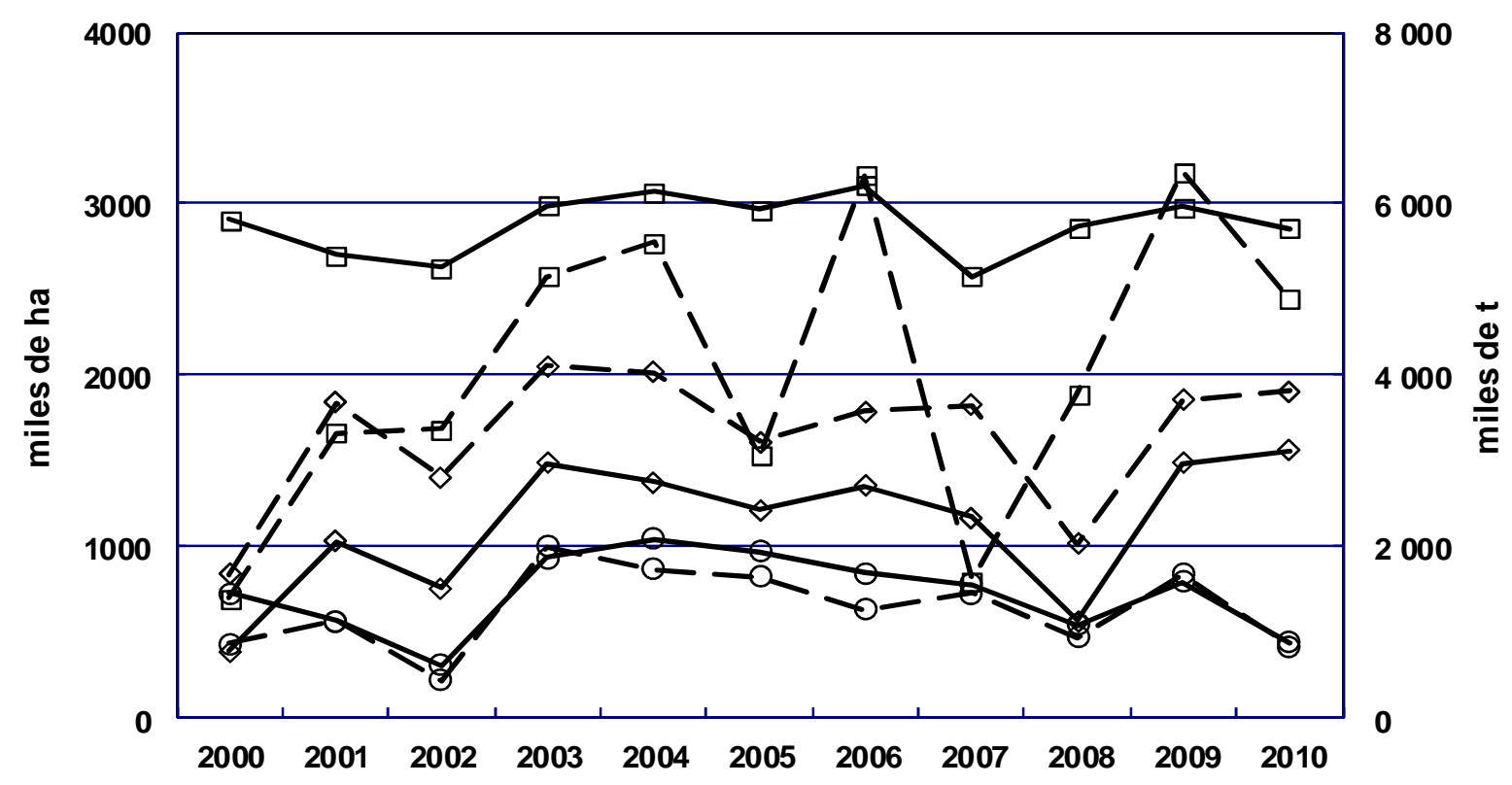

En la imagen queda de manifiesto la importancia de la superficie de cultivo de trigo en Marruecos, con valores bastante estables que se aproximan a los 3 millones de ha, y que algunos años los superan. En Argelia la superficie ha evolucionado de forma variable en el periodo considerado, aunque con una tendencia global creciente. Las superficies de Túnez, también variables, son lógicamente menores. Las tendencias de la producción son distintas. La producción total en Argelia guarda un paralelismo bastante claro con las superficies cultivadas cada año, y lo mismo ocurre en Túnez. Sin embargo, la cantidad de trigo producido en Marruecos muestra unas variaciones muy notables, distintas a las de superficie.

En cuanto al rendimiento, los valores correspondientes quedan reflejados en el Gráfico 2 , que se muestra a continuación. 
Gráfico 2. Evolución del rendimiento medio del cultivo de trigo en Argelia $(\diamond)$, Marruecos ( $\square$ ) y Túnez (०) durante los últimos años

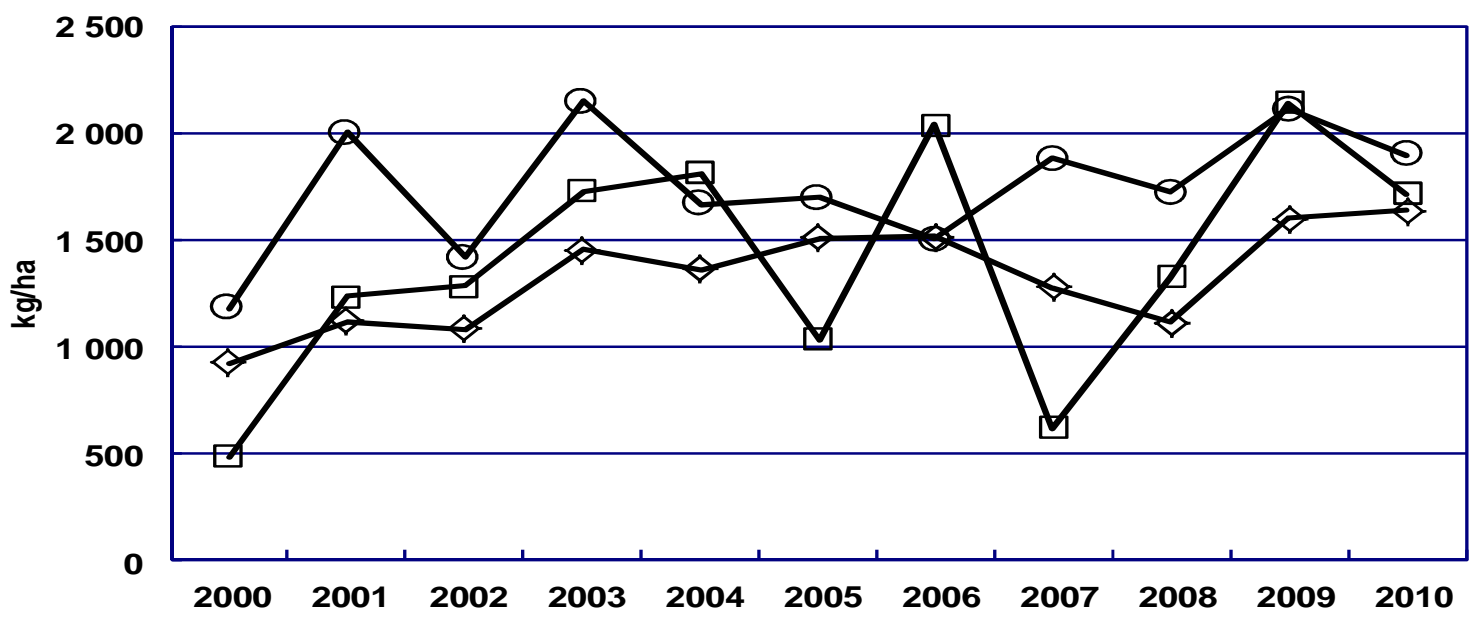

Los mayores rendimientos corresponden a Túnez, y los menores, en términos globales, a Argelia. Se observa que los rendimientos son bastante variables, sobre todo en el caso de Marruecos; esta variabilidad es la responsable de las oscilaciones en el valor de la producción final de este país.

En lo relativo a la cebada, los datos de superficie y producción de los tres países a lo largo de los últimos años quedan reflejados en el gráfico adjunto.

Gráfico 3. Evolución de la superficie y producción del cultivo de cebada en Argelia $(\diamond)$, Marruecos ( $\square$ ) y Túnez (०) durante los últimos años. Superficie, línea continua; producción, línea de trazos

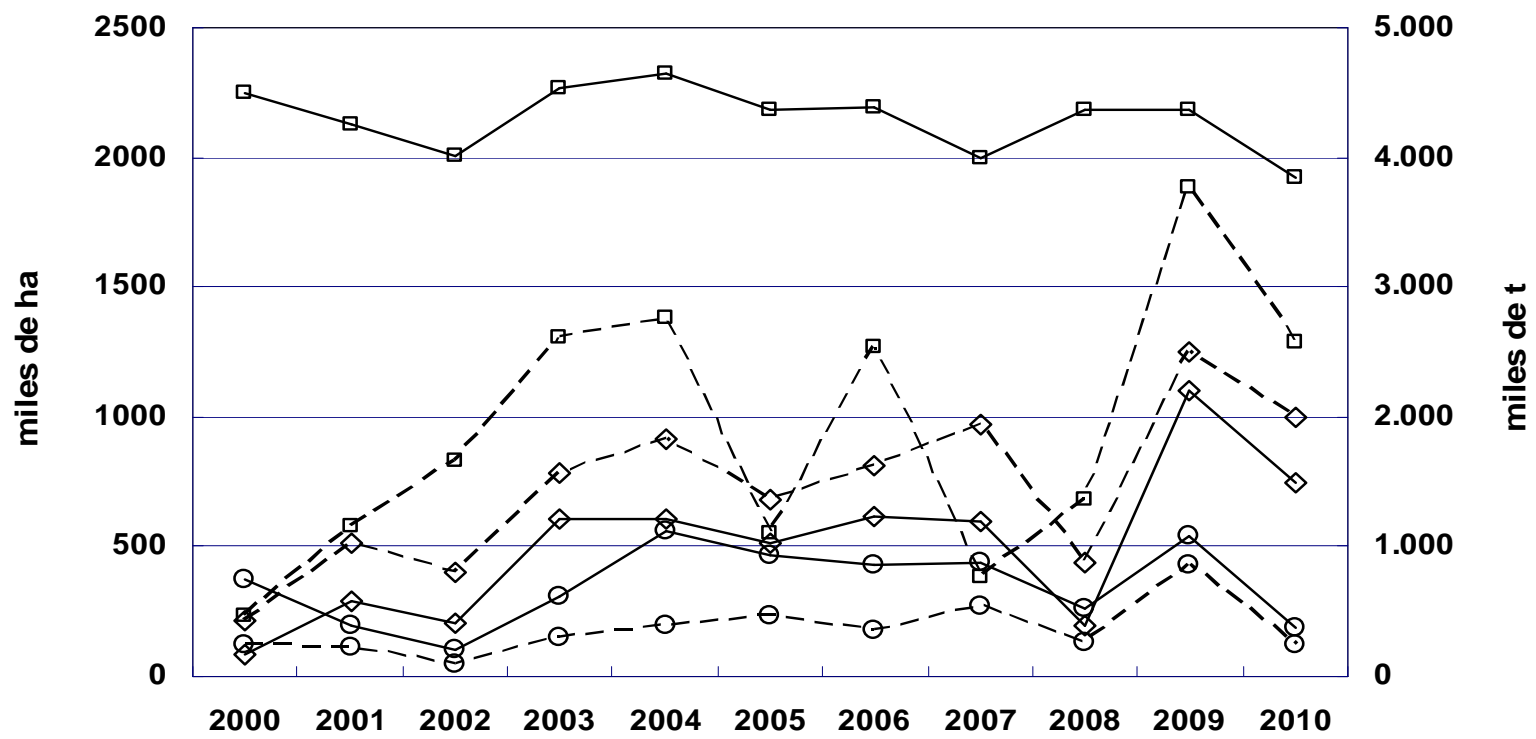


Queda de manifiesto la gran diferencia de superficie cultivada entre Marruecos por un lado y Argelia y Túnez por el otro. Sin embargo, al igual que ocurría con el trigo, la producción final de Marruecos presenta más oscilaciones, y en algunos años su producción final es del mismo orden que la de los otros dos países, a pesar de que la superficie dedicada sea al menos el doble.

Los datos correspondientes a los rendimientos medios quedan reflejados en el Gráfico 4. Al final del periodo analizado los valores en los distintos países son muy similares; la tendencia global es ligeramente creciente en el caso de Argelia, y más acusada en Marruecos y Túnez, que presentan datos más variables previamente.

Gráfico 4. Evolución del rendimiento medio del cultivo de cebada en Argelia $(\diamond)$, Marruecos ( $\square$ ) y Túnez ( $\odot$ ) durante los últimos años

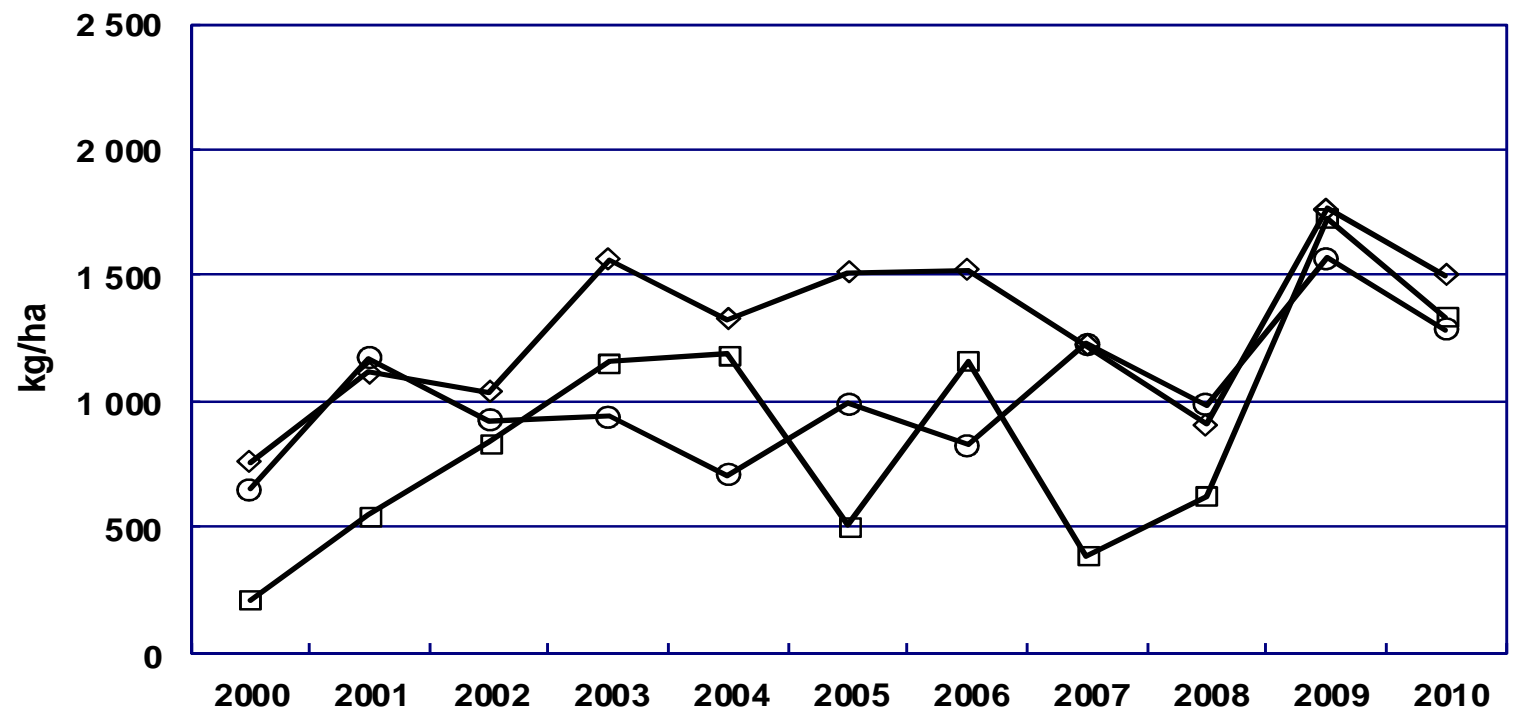

Por otra parte, los datos de superficie y producción correspondientes al cultivo del olivar se reflejan a continuación, en el Gráfico 5 adjunto: 
Gráfico 5. Evolución de la superficie y producción del cultivo de olivo en Argelia $(\diamond)$, Marruecos ( $\square$ ) y Túnez $(\circ)$ durante los últimos años. Superficie, línea continua; producción, línea de trazos.

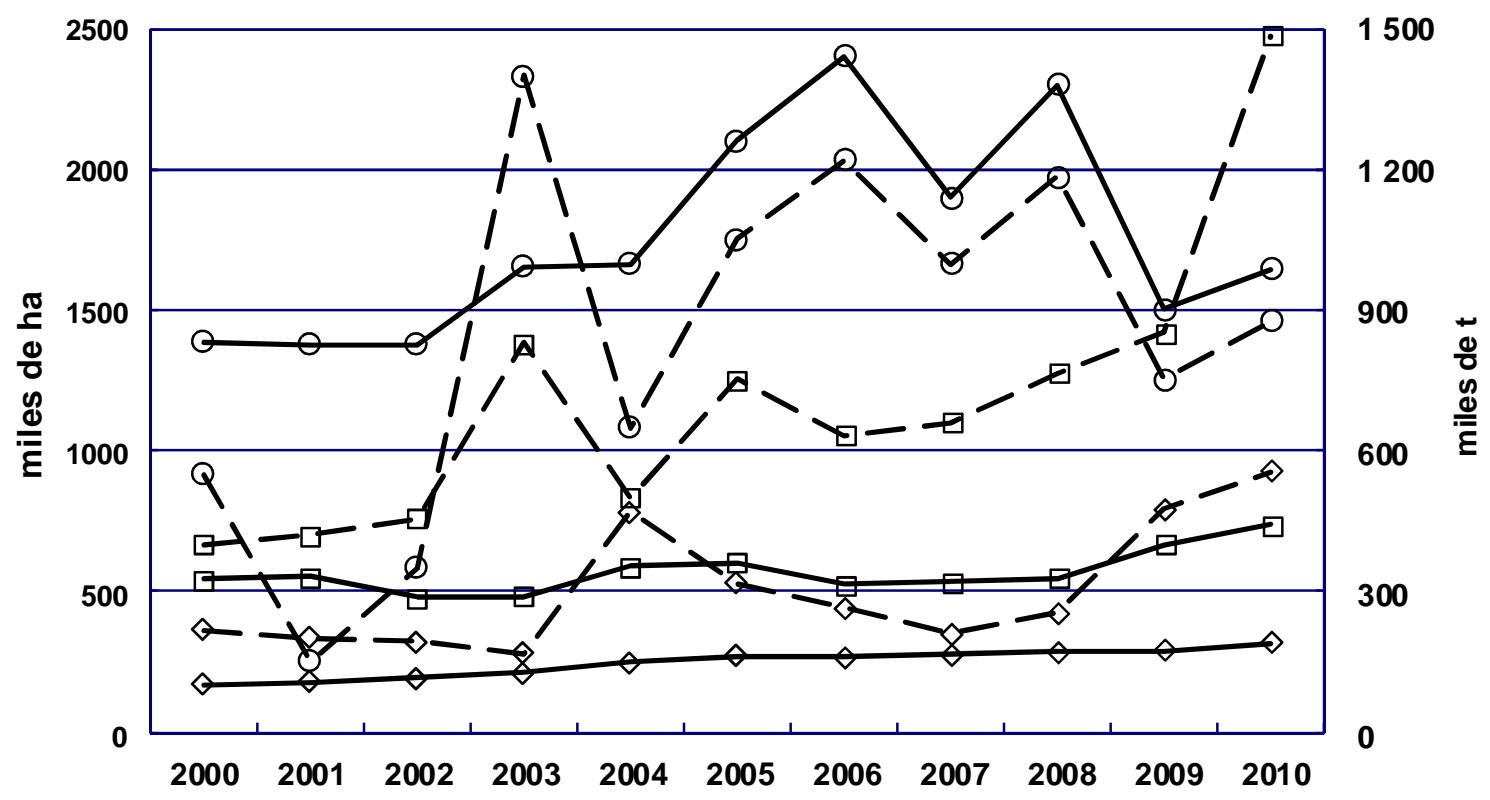

Se puede observar que, en general, existe un paralelismo bastante grande entre superficie y producción. Sin embargo, hay diferentes cuestiones a considerar; por una parte, la variabilidad entre campañas de la superficie dedicada a olivar en Túnez. Al tratarse de un cultivo arbóreo, esa variabilidad no tiene una justificación razonable, sobre todo en términos de aumento de un año al siguiente, salvo que lo que se esté computando es la superficie realmente cosechada del cultivo, y no la total.

También es de destacar el aumento notable de producción en los últimos años en Argelia y, sobre todo, en Marruecos.

La evolución del rendimiento medio en estos tres países está recogida en el Gráfico 6 siguiente. 
Gráfico 6. Evolución del rendimiento medio del cultivo de olivo en Argelia $(\diamond)$, Marruecos ( $\square)$ y Túnez ( $\odot$ ) durante los últimos años.

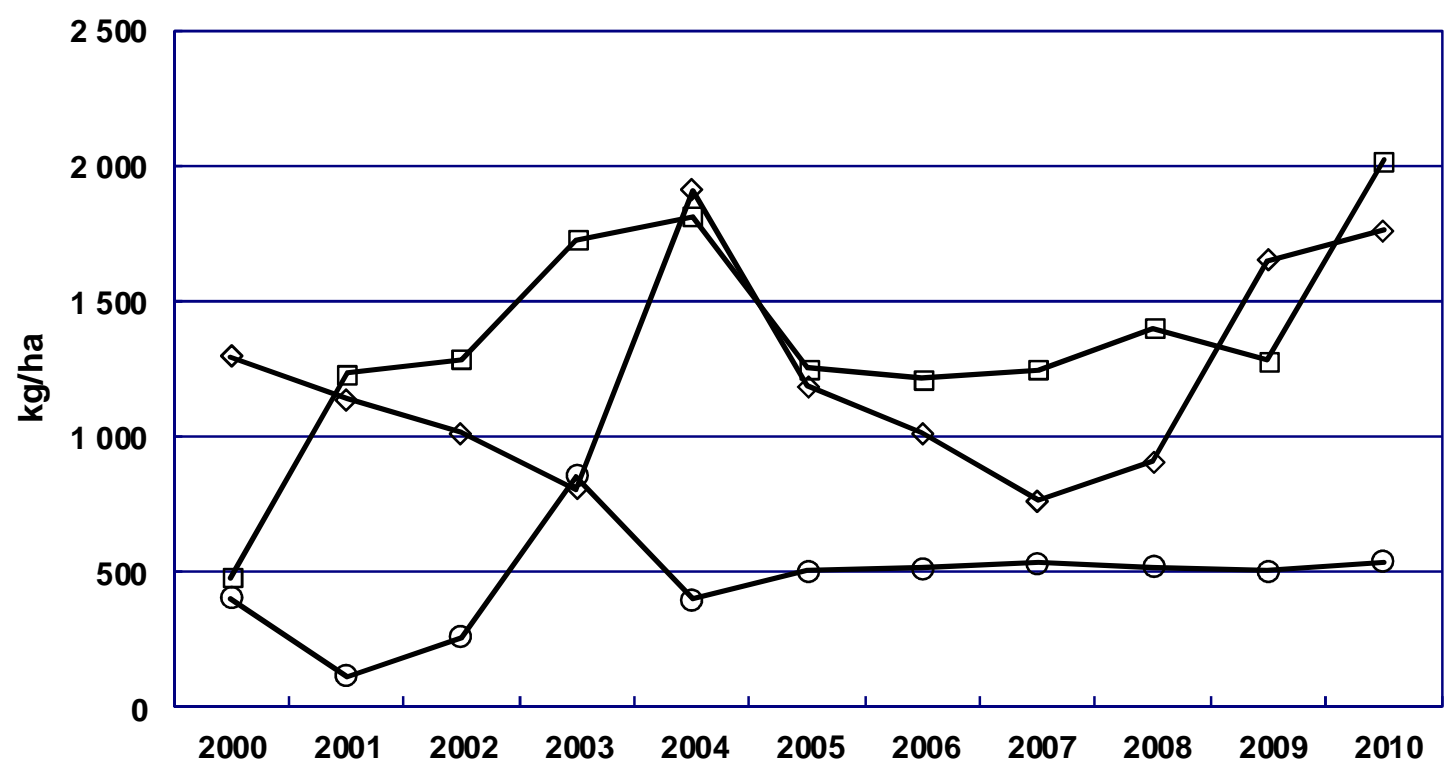

En esta gráfica se constata, por una parte, la progresión en los rendimientos en Argelia y Marruecos, que contribuye a explicar el aumento de producción en estos países. Por otro lado, el mantenimiento casi invariable del rendimiento en Túnez puede confirmar la hipótesis de que la superficie que se contabiliza en las estadísticas (al menos para este país) es la cosechada en cada año.

\subsection{Producción ganadera}

En el análisis de la producción ganadera conviene considerar tanto las características de la cabaña de cada especie como la producción o producciones que esta cabaña proporciona. En la faceta de ganadería disponible, el Gráfico 7 muestra la evolución en los tres países de los censos de ganado ovino y caprino. Los datos proceden de las estadísticas de FAO, tanto en lo relativo a poblaciones de las diferentes especies ${ }^{14}$ como a las producciones obtenidas ${ }^{15}$.

\footnotetext{
${ }^{14}$ FAO (2012): Base de Datos FAOSTAT, Estadísticas de censos ganaderos: http://faostat.fao.org/site/573/ default.aspx\#ancor.

${ }^{15}$ FAO (2012): Base de Datos FAOSTAT, Estadísticas de producciones animales: http://faostat.fao.org/site/569/ default.aspx \#ancor.
} 
Gráfico 7. Evolución e importancia del censo de ganado ovino y caprino en Argelia $(\diamond)$, Marruecos $(\square)$ y Túnez $(\odot)$ durante los últimos años. Ganado ovino, línea continua; ganado caprino, línea de trazos

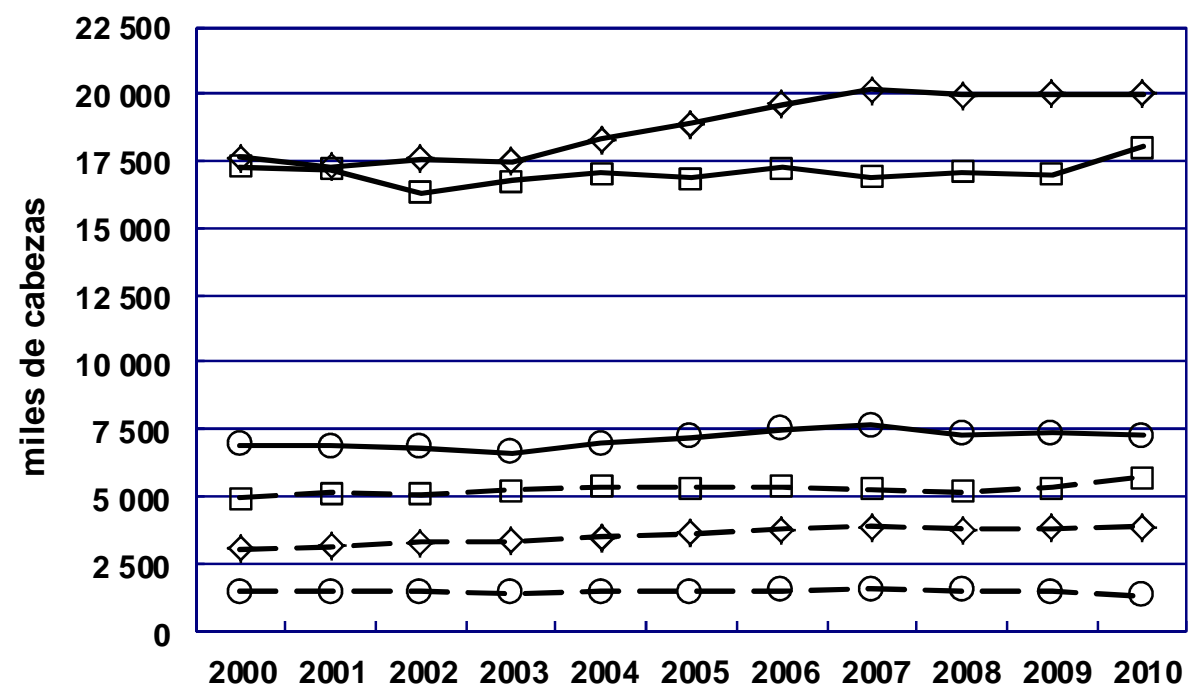

En los tres países hay un predominio claro de ovino sobre caprino, si bien en el caso de Argelia la importancia relativa, en dimensión de la cabaña, del ganado caprino es algo menor. En los tres casos los censos se mantienen bastante estables, con una ligera tendencia creciente más o menos apreciable.

El ganado vacuno, aunque sea numéricamente menos importante, es una especie de gran relevancia en el suministro de producciones animales. La cabaña de esta especie en los tres países, y su evolución en los últimos años, se muestran en el Gráfico 8. 
Gráfico 8. Evolución e importancia del censo de ganado vacuno en Argelia $(\diamond)$, Marruecos ( $\square$ ) y Túnez ( $\odot$ ) durante los últimos años

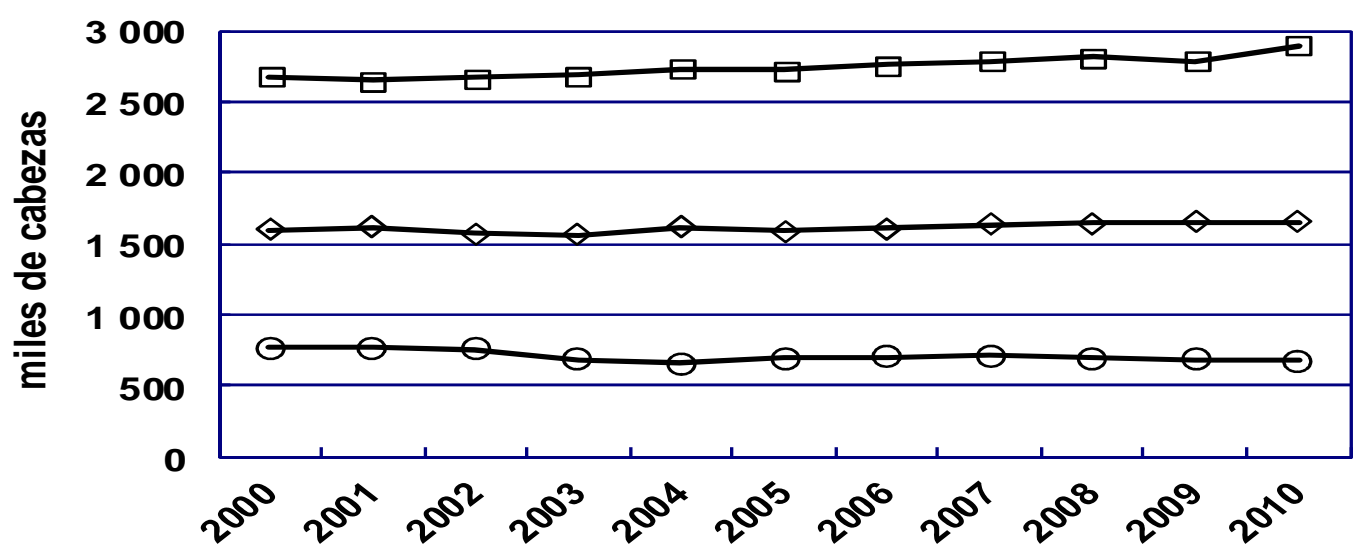

La tendencia de los censos de esta especie es de una gran estabilidad, aunque se detecte una cierta disminución en el caso de Túnez. Llama la atención la gran diferencia de tamaño entre las cabañas de Marruecos y Argelia.

Las especies equinas tienen una presencia desigual en estos tres países. Los datos correspondientes quedan reflejados en el Gráfico 9.

Gráfico 9. Evolución e importancia de los censos de equinos en Argelia ( $\diamond)$, Marruecos ( $\square)$ y Túnez (०) durante los últimos años. Ganado caballar, línea continua; ganado asnal, línea de trazos; ganado mular, línea de puntos.

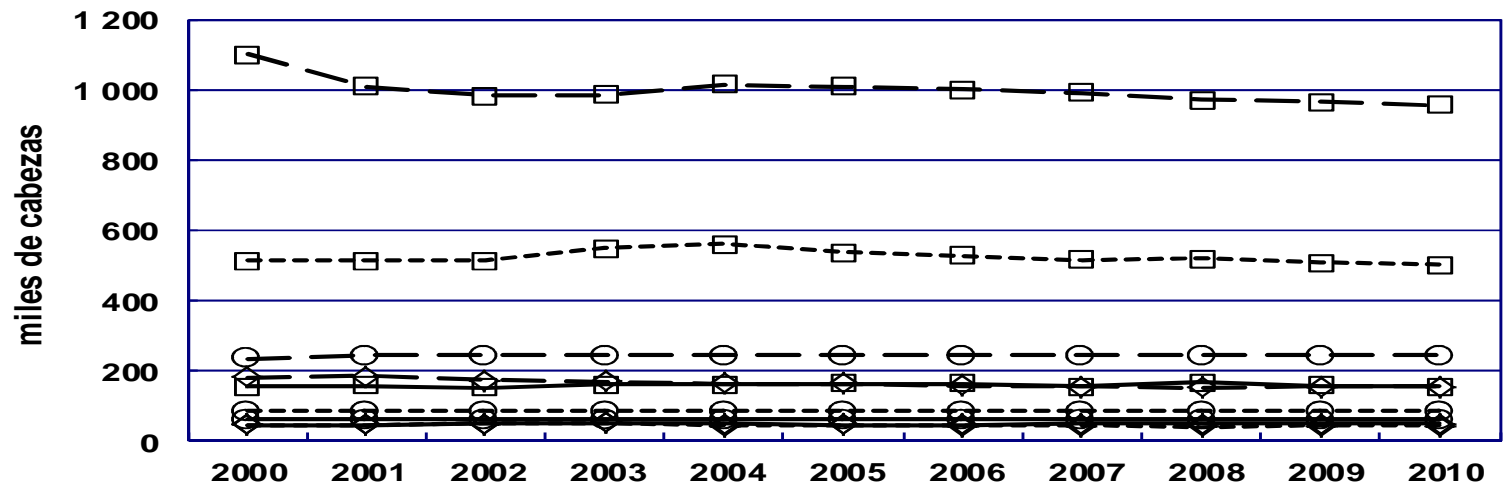


También en este caso son destacados los datos correspondientes a Marruecos que, por otra parte, muestran bastante estabilidad. La población asnal (y en menor medida, mular) de este país es notablemente superior a la de Argelia y Túnez, lo que puede ser indicio de una menor mecanización y de la conservación e implantación de otras alternativas de tracción y transporte. La causa puede radicar en un grado objetivamente menor de desarrollo, o bien en la mejor adaptación a condiciones de terreno muy poco aptas para los medios mecánicos. Las poblaciones menores, con diferencia, en estas especies corresponden a Argelia.

Para completar las especies con presencia relevante, se recogen en el Gráfico 10 las poblaciones de gallinas y de camellos. Los censos de gallinas en Argelia y Marruecos son del mismo orden de magnitud, aunque el de Marruecos muestra una tendencia claramente creciente. También tiene esta tendencia la población en Túnez. En cuanto a los camellos, resulta especialmente destacado el censo de Túnez, que no tiene una justificación aparente clara, y llama asimismo la atención a primera vista la escasa cuantía de esta especie en Marruecos, que puede explicarse por la menor importancia relativa de las zonas desérticas y subdesérticas en su territorio.

Gráfico 10. Evolución e importancia del censo de gallinas y camélidos en Argelia $(\diamond)$, Marruecos ( $\square$ ) y Túnez $(\circ)$ durante los últimos años. Gallinas, línea continua; camellos, línea de trazos

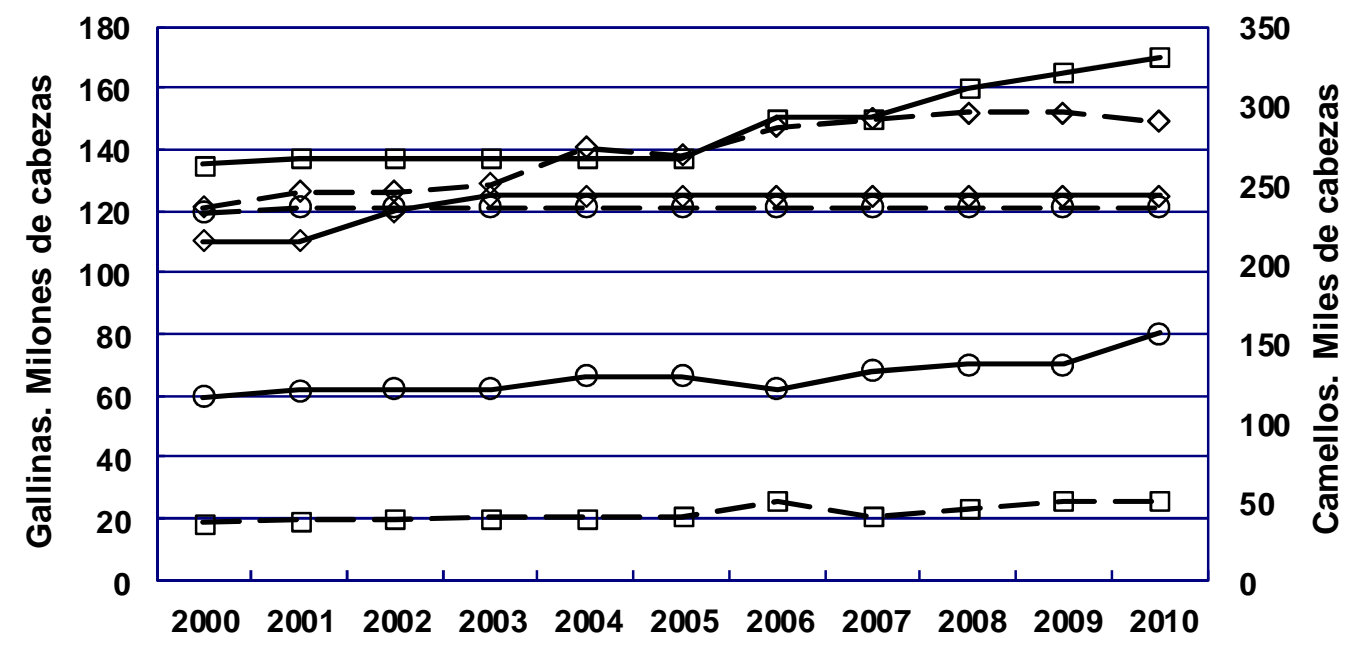

Al tratarse de países esencialmente musulmanes, la cabaña porcina es puramente testimonial (el máximo registrado es en Marruecos, con menos de 9.000 cabezas), y en todos ellos se pueden encontrar conejos, patos, pavos, gallinas de Guinea, etc. en cantidades variables. 
En lo que se refiere a las producciones ganaderas, el Gráfico 11 muestra en primer lugar la producción de leche de oveja y cabra en cada uno de los tres países durante los últimos años.

Gráfico 11. Evolución e importancia de la producción de leche en Argelia ( $\diamond)$, Marruecos (๑) y Túnez ( $\odot$ ) durante los últimos años. Leche de oveja, línea continua; leche de cabra, línea de trazos

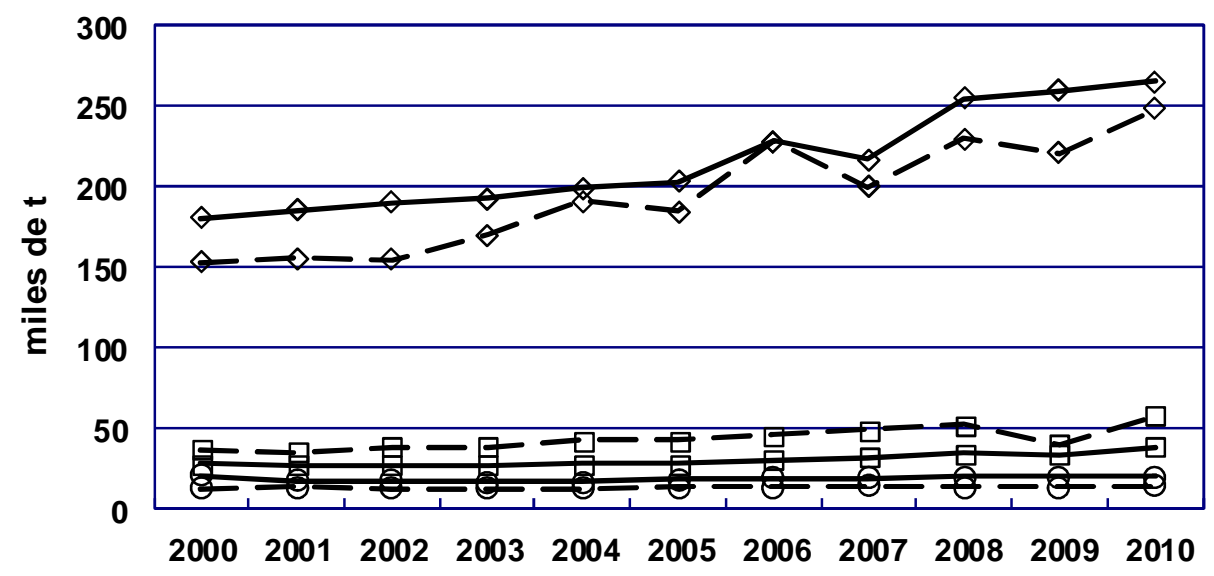

Se observa que en los tres países la producción de leche de ambas especies es del mismo orden de magnitud. Sin embargo, destaca significativamente que la producción de leche en Marruecos es muy inferior, en relación a la dimensión de las respectivas cabañas, que la de los otros dos países. En todos los casos, la comparación de estas producciones con los respectivos censos indica claramente que la productividad láctea del ganado caprino es mucho más alta que la del ovino.

El otro gran apartado de producción láctea corresponde a la leche de vacuno. En el Gráfico 12 se muestra la evolución de esta producción en los tres países. Se ha incluido asimismo la producción de leche de camella, a escala distinta. 
Gráfico 12. Evolución e importancia de la producción de leche en Argelia $(\diamond)$, Marruecos $(\square)$ y Túnez $(\circ)$ durante los últimos años. Leche de vaca, línea continua; leche de camella, línea de trazos

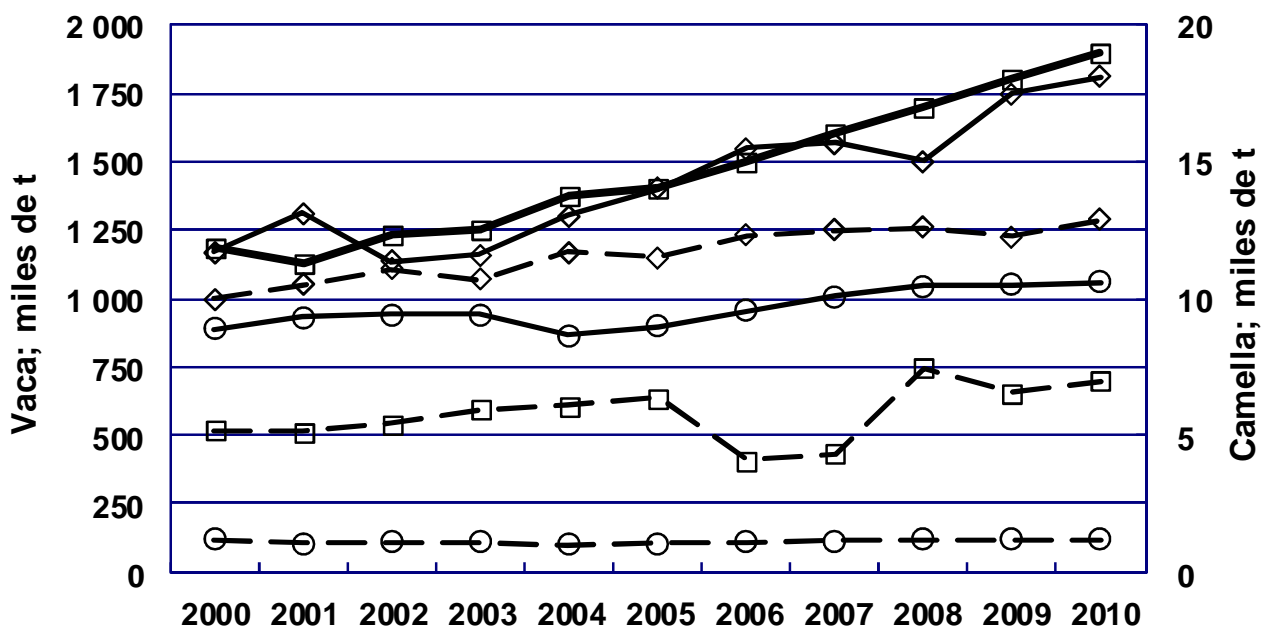

En el caso de la leche de vacuno, la productividad es muy superior en Argelia; la población vacuna es prácticamente la mitad que en Marruecos, y se alcanzan producciones globales similares. En este aspecto influyen numerosos factores, desde la estructura y pureza racial de la cabaña hasta los sistemas de explotación y alimentación. En todo caso, tanto en Marruecos como en Argelia se observa una tendencia creciente de la producción a lo largo del periodo mostrado, con aumentos del 40-50\%.

En Túnez también se ha producido un aumento, pero mucho menor. La leche de camella tiene unas producciones bastante reducidas, con cifras máximas en Argelia.

La producción de carne se muestra en las gráficas siguientes. En primer lugar figura la correspondiente a ovino y caprino, aunque cuantitativamente no sean las más importantes. 
Gráfico 13. Evolución e importancia de la producción de carne en Argelia ( () , Marruecos ( $\square$ ) y Túnez ( $\odot$ ) durante los últimos años. Carne de ovino, línea continua; carne de caprino, línea de trazos

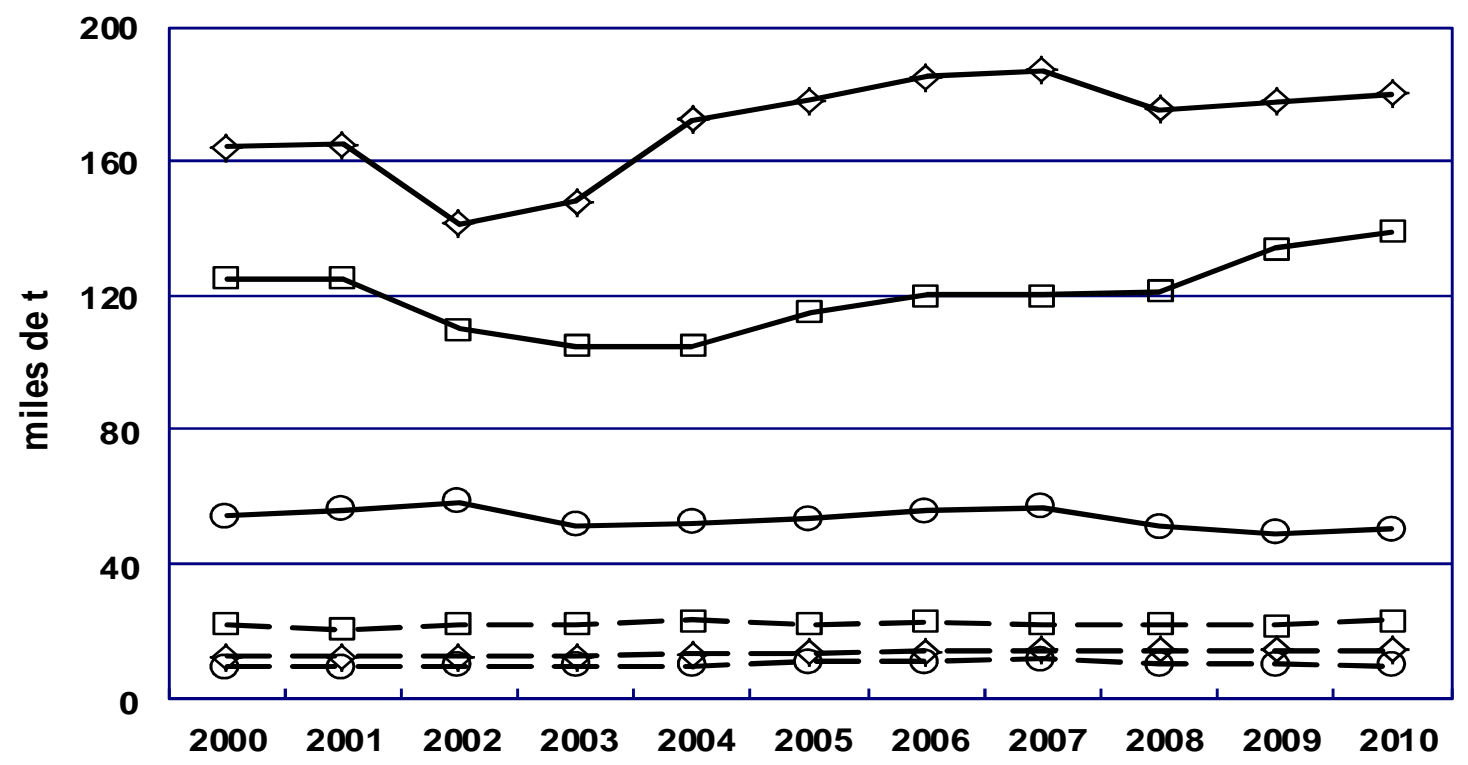

En el caso de la carne de ovino, la producción argelina es claramente superior a la de Marruecos, a pesar de que las cabañas son del mismo orden de magnitud; hay que recordar, además, que la producción láctea también es superior en Argelia. Eso puede indicar una mayor tecnificación e intensificación de esta ganadería. La carne de caprino tiene en los tres países una importancia mucho menor, y guarda una mejor concordancia (aunque no completa) con la dimensión de los respectivos censos.

Las otras dos grandes producciones cárnicas son las relativas a vacuno y ave, concretamente pollo. El Gráfico 14 recoge los datos correspondientes. 
Gráfico 14. Evolución e importancia de la producción de carne en Argelia $(\diamond)$, Marruecos $(\square)$ y Túnez $(\circ)$ durante los últimos años. Carne de vacuno, línea continua; carne de pollo, línea de trazos.

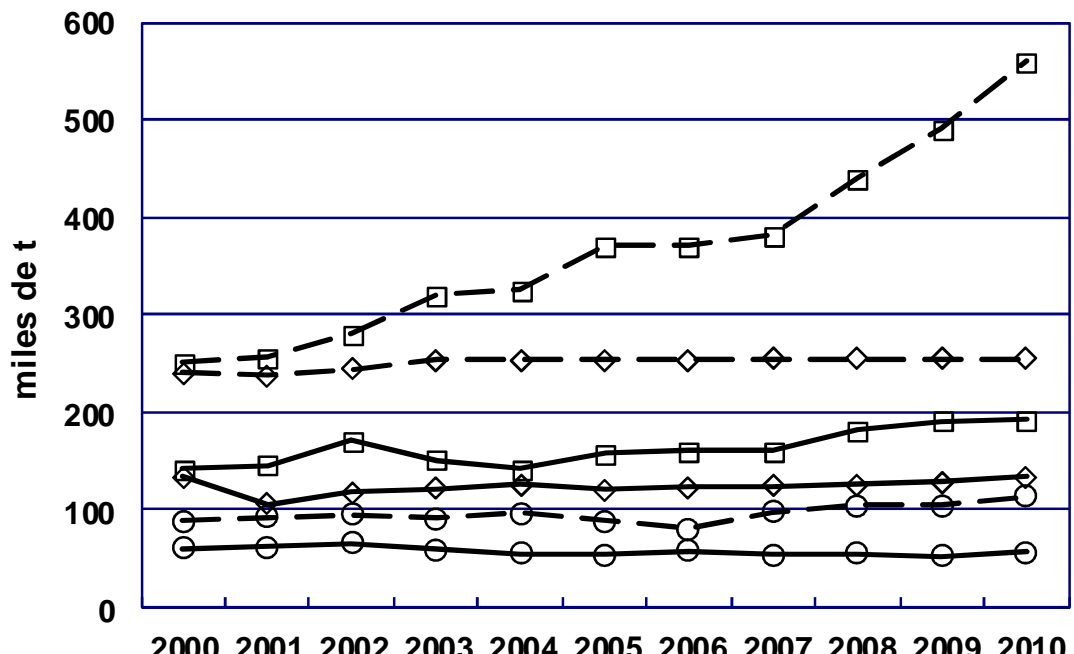

La producción de carne de vacuno es mayor en Marruecos; ha ido progresando paulatinamente. Sin embargo, la producción de carne por cabeza es menor que en los otros dos países. En el caso de la carne de pollo, tanto Argelia como Túnez mantienen un ritmo global de producción estable, mientras que la producción en Marruecos es superior y creciente. Además, mientras que la productividad en Marruecos es máxima y ha crecido más que la cabaña correspondiente, en el caso de Argelia la cantidad de carne por unidad de censo se ha reducido, ya que la producción está estabilizada y el censo ha ido aumentando paulatinamente.

Para completar la perspectiva de producciones ganaderas, se pueden revisar los datos correspondientes a la producción de huevos. El Gráfico 15 muestra los datos relativos a la producción de huevos de gallina, que es la más importante con gran diferencia.

La mayor producción corresponde a Marruecos, aunque se ha reducido apreciablemente a partir de 2008. Esta evolución no concuerda bien con la tendencia progresiva y creciente del censo. La producción en Argelia se incrementó notablemente en 2002, y desde entonces sigue aumentando paulatinamente. Las cifras de Túnez están completamente estabilizadas. 
Gráfico 15. Evolución e importancia de la producción de huevos de gallina en Argelia $(\diamond)$, Marruecos ( $\square)$ y Túnez $(\odot)$ durante los últimos años



\section{Algunas consideraciones sobre el potencial de crecimiento de la producción agraria}

En este punto se realizará un análisis global de las posibilidades de crecimiento de la producción agraria en los países considerados, teniendo en cuenta esencialmente a algunos de los factores que pueden ser indicativos de las restricciones actuales y futuras para ese potencial.

En el marco del medio físico, el relieve del terreno y las cualidades del suelo son dos elementos determinantes. Aunque es suficientemente conocida en líneas generales, la topografía de la zona queda reflejada básicamente en la imagen adjunta. 


\section{Figura 2. Representación genérica del relieve topográfico en el Magreb Central}

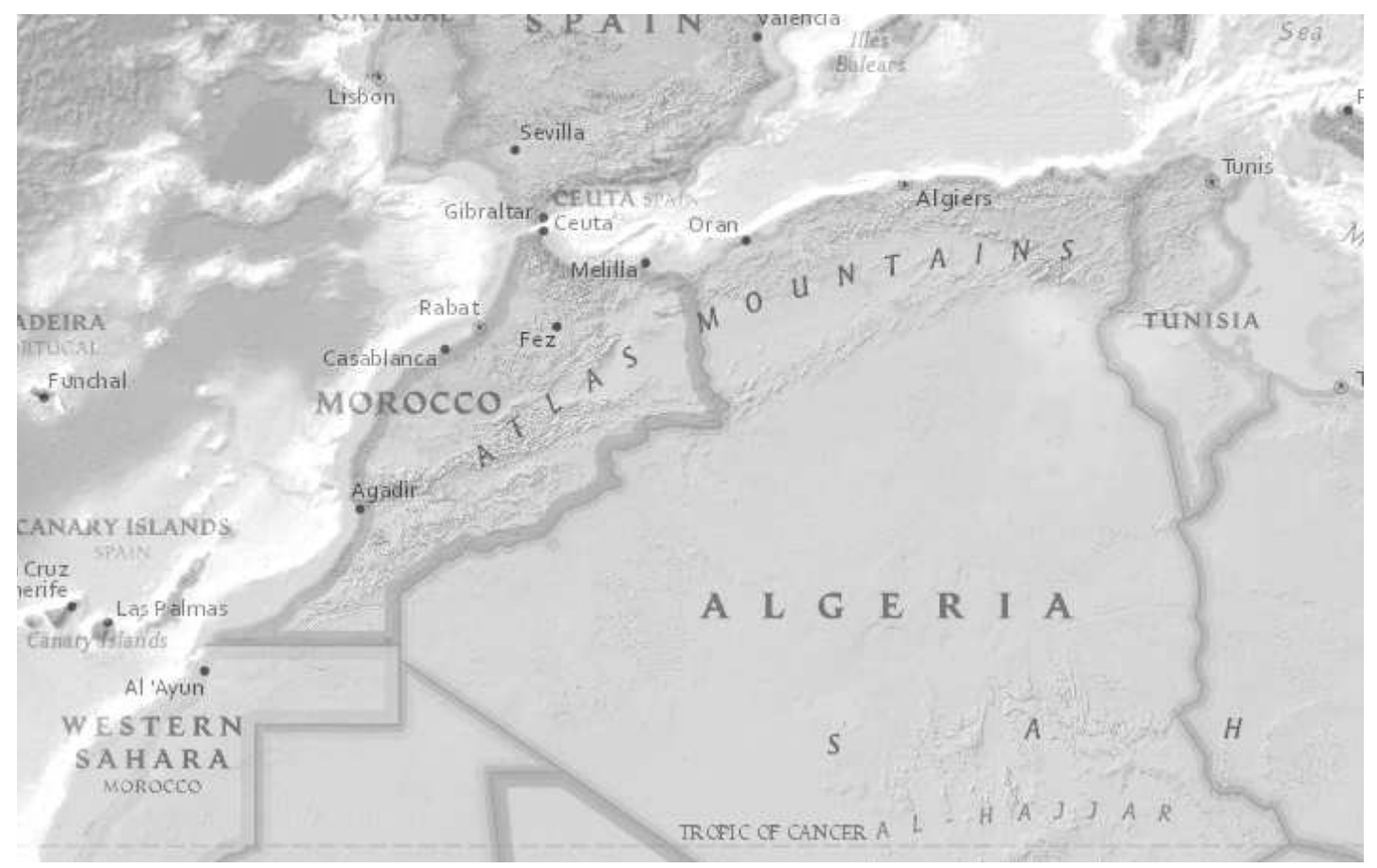

Base cartográfica: National Geographic Society ${ }^{16}$

En los tres países, las zonas más dedicadas a la actividad agrícola y ganadera se caracterizan, en términos generales, por una orografía acentuada, que tiene su máxima expresión en las montañas del Atlas, tanto en Marruecos como en su extensión hacia el este en las dos ramas del Tell (Tell costero y Tell sahariano) y en su extremo oriental en la Kabilia y otros macizos montañosos en la frontera entre Argelia y Túnez y en la zona norte de este último país. Estas condiciones de relieve restringen las posibilidades de un cultivo extensivo con un grado elevado de mecanización. Por otro lado, estas mismas condiciones dificultan la formación de suelos muy evolucionados y con alta fertilidad. Fuera de esas zonas, las superficies más suaves corresponden, en su mayor parte, a áreas donde se imponen otros factores restrictivos, sobre todo la falta de agua y las temperaturas extremas.

Otro aspecto a considerar es el ya apuntado de la disponibilidad de agua. En lo relativo a la pluviometría, ya se han comentado al inicio de este documento algunas características básicas de esta disponibilidad, en términos de pluviometría (Figura 1).

Otra forma de considerar la repercusión del régimen pluviométrico es el "índice de aridez", representado aquí por el cociente entre la precipitación media anual y la evapotranspiración de referencia. En la Figura 3, las áreas costeras y la pequeña mancha de las cumbres del Atlas, oscuras, corresponden a cocientes superiores a 0,65 (climas húmedos); las zonas contiguas ligeramente más claras representan climas secos-subhúmedos (cociente entre 0,5 y 0,65 ). El tono más claro representa las áreas con climas semiáridos (cociente entre $0,2$ y 0,5$)$; los tonos más oscuros hacia el sur corresponden a climas áridos o hiper-áridos.

\footnotetext{
${ }^{16}$ National Geographic Map Maker, en http://educationlnatinalgeographic.com/education/mapping/.
} 
Figura 3. Representación gráfica del índice de aridez (relación entre pluviometría y evapotranspiración potencial). Explicación en el texto

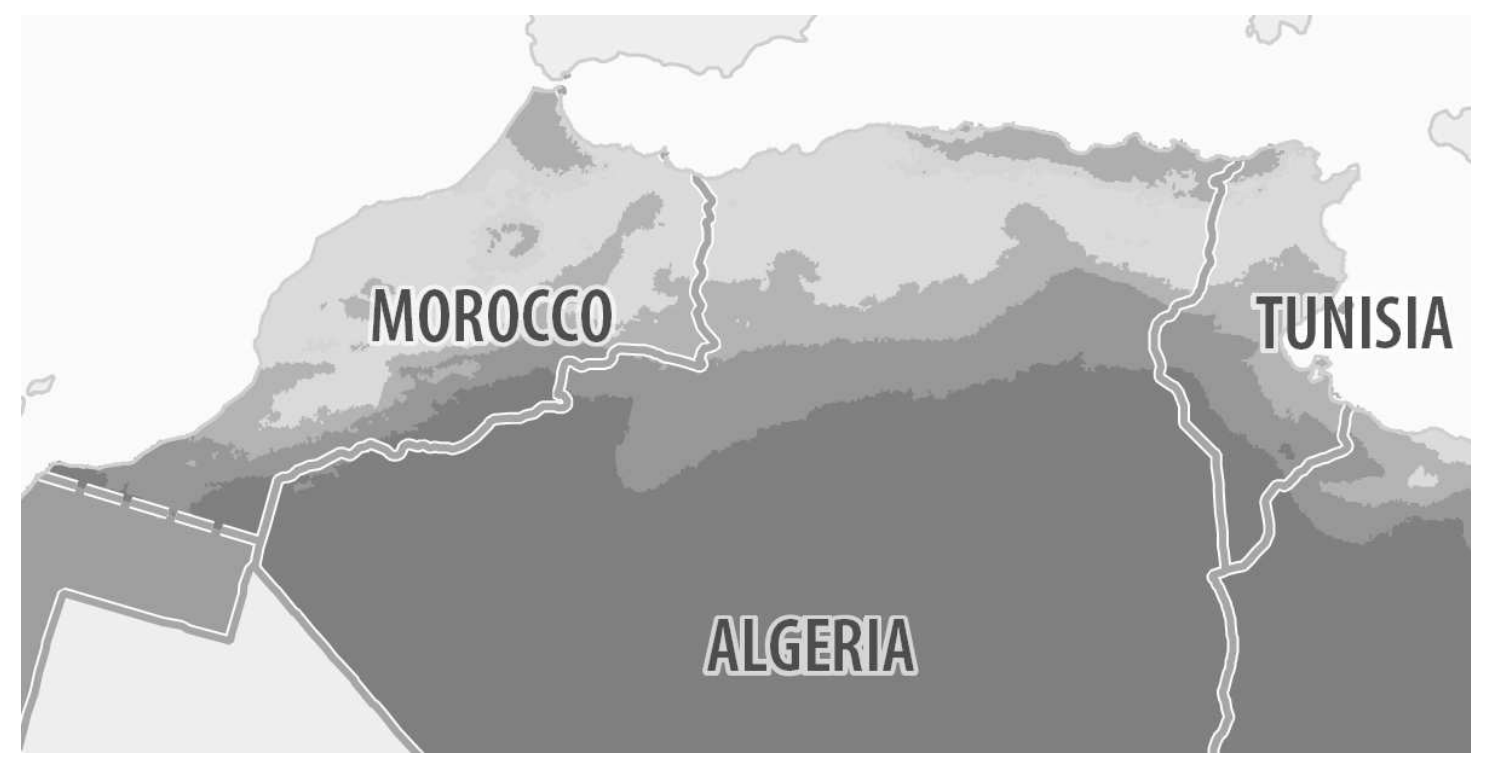

Fuente: The World Bank (2007) ${ }^{17}$

Las posibilidades de uso de agua en la agricultura vienen asimismo indicadas por la cuantía de los recursos hídricos disponibles. La Tabla 8 adjunta refleja las cantidades más significativas en este aspecto en los tres países del área. También se incluyen, como referencia, los valores correspondientes a España.

Tabla 8. Disponibilidad de agua en los países del Magreb Central y en España

\begin{tabular}{|l|c|c|c|c|}
\hline \multicolumn{1}{|c|}{ País } & $\begin{array}{c}\text { Recursos hídricos } \\
\text { renovables totales } \\
\left(\mathrm{km}^{3} / \text { año }\right)\end{array}$ & $\begin{array}{c}\text { Uso, como } \\
\text { porcentaje de } \\
\text { los recursos }\end{array}$ & $\begin{array}{c}\text { Recursos hídricos } \\
\text { renovables per cápita } \\
(2011)\left(\mathrm{m}^{3} / \mathrm{hab} \cdot \mathrm{año}\right)\end{array}$ & $\begin{array}{c}\text { Capacidad de } \\
\text { embalses } \\
\left(\mathrm{km}^{3}\right)^{*}\end{array}$ \\
\hline Argelia & 11,7 & 55 & 324,3 & 5,7 \\
Marruecos & 29,0 & 40 & 898,6 & 16,9 \\
Túnez & 4,6 & 60 & 433,7 & 2,5 \\
\hline España & 111,5 & 30 & $2.400,0$ & 52,6 \\
\hline
\end{tabular}

* Datos de 2008, excepto España (2010)

Fuente: Aquastat, $\mathrm{FAO}^{18}$

\footnotetext{
${ }^{17}$ Bucknall, Julia (ed.) (2007): "Making the most of scarcity: Accountability for better water management results in the Middle East and North Africa", MENA development report, Washington D.C. - The World Bank, en http://documents.worldbank.org/curated/en/2007/01/8468587/making-most-scarcity-accountability-better-watermanagement-results-middle-east-north-africa.
} 
Esta Tabla muestra una serie de datos de gran interés. El primero de ellos, la escasa cuantía de los recursos hídricos renovables en la zona, derivada tanto de la reducida pluviometría a la que se ha aludido como de la ausencia de infraestructuras que permitan su regulación. Más adelante se señalarán algunos hechos en este último sentido. Por otro lado, se puede observar que el uso que se realiza de los recursos es bastante elevado, razón por la cual la intensidad del uso está en grados que resultan difíciles de aumentar. La cifra de recursos renovables per cápita es especialmente ilustrativa. El umbral internacional de escasez de agua se cifra en los $1.000 \mathrm{~m}^{3}$ por habitante y año, de modo que los tres países considerados están en condiciones de escasez. En el caso de Marruecos, la cifra límite se ha alcanzado en épocas crecientes, a medida que la población se ha ido incrementando. En Argelia y Túnez la situación es más dramática.

Por último, la capacidad de regulación mediante embalses es claramente reducida, aunque también en este aspecto hay diferencias notables. Por otro lado, tanto Argelia como Marruecos han iniciado en los últimos años ambiciosos programas de construcción de embalses y otras infraestructuras mediante los cuales se pretende aumentar significativamente la disponibilidad de agua.

El grado de tecnificación de la agricultura y, por tanto, sus posibilidades de incremento de producción, pueden estimarse mediante el análisis de algunos indicadores que reflejen el uso de diferentes inputs y medios de producción. Uno de ellos es el consumo de nutrientes vegetales por la agricultura. La evolución de los datos correspondientes se muestra, para los tres países, en el Gráfico 16 adjunto.

\footnotetext{
${ }^{18}$ FAO (2012): Base de Datos AQUASTAT, Hojas de datos nacionales.
} 
Gráfico 16. Consumo aparente de macronutrientes de los cultivos en Argelia $(\diamond)$, Marruecos ( $\square$ ) y Túnez ( $($ ) durante los últimos años. Líneas continuas, consumo de nitrógeno; líneas de trazos, consumo de fósforo; líneas de puntos, consumo de potasio

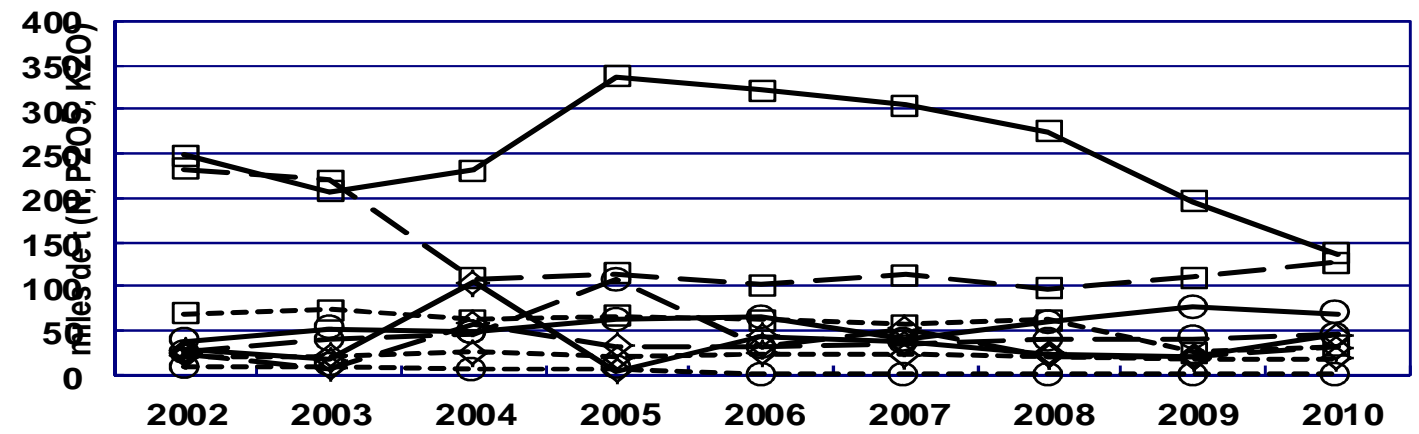

Fuente: Faostat, FAO $^{19}$

Como dato básico general, llama la atención el escaso consumo de nutrientes en la agricultura argelina, con cifras inferiores en algunos casos a las correspondientes a Túnez. Por otro lado, hay una variabilidad bastante notable en la evolución del consumo de nitrógeno en Marruecos, que no tiene una explicación fácil. Un tercer punto llamativo es que, según las estadísticas manejadas, el consumo de potasio como nutriente en Túnez es nulo desde el año 2006 en adelante.

Para disponer de una referencia en cuanto al grado de uso de fertilizantes minerales en la agricultura de cada uno de estos países, que es uno de los más importantes indicadores de tecnificación, se ha elaborado la Tabla 9 que sigue. En la aportación de los profesores Soler y Arroyo también hay un interesante análisis de estos indicadores de tecnificación. Como elemento de orientación, se han incluido los datos correspondientes a otros tres países mediterráneos, España, Israel e Italia.

\footnotetext{
${ }^{19}$ FAO (2012): Base de Datos FAOSTAT, Estadísticas de fertilizantes, en http://faostat.fao.org/site/575/default.aspx \#ancor.
} 
Tabla 9. Consumo aparente estimado de nutrientes en diferentes países del área mediterránea

\begin{tabular}{|l|c|c|c|}
\hline \multirow{2}{*}{ País } & \multicolumn{2}{|c|}{ Kg de elemento / ha de superficie cultivada } \\
\hline & $\mathrm{N}$ & $\mathrm{P}_{2} \mathrm{O}_{5}$ & $\mathrm{~K}_{2} \mathrm{O}$ \\
\hline Argelia & 4,7 & 4,2 & 4,2 \\
Marruecos & 27,2 & 15,2 & 8,5 \\
Túnez & 21,2 & 11,4 & 0,0 \\
España & 61,6 & 20,8 & 13,1 \\
Israel & 134,5 & 23,1 & 69,7 \\
Italia & 68,2 & 23,2 & 21,4 \\
\hline
\end{tabular}

Estimación a partir de datos de FAO, $2009^{20}$

Es evidente que pueden y deben existir diferencias entre países en función de los sistemas de cultivo (secano, regadío, cultivo extensivo mecanizado, ...) y de las especies que se desarrollan; no tienen las mismas demandas de nutrientes los cultivos hortícolas de primor que los cereales en secano. Sin embargo, las diferencias entre Argelia (sobre todo), Marruecos y Túnez por una parte y los otros tres países por el otro son patentes, e indican a las claras que existe un potencial de progreso en productividad evidente, aunque esté limitado por otros aspectos como la climatología.

Otro aspecto del grado de tecnificación que se contempla en la aportación de los profesores Soler y Arroyo se refiere al uso de maquinaria. En la Tabla 10 siguiente se muestran algunos datos al respecto.

\footnotetext{
${ }^{20}$ FAO (2012): Base de datos FAOSTAT, Estadísticas de fertilizantes .
} 
Tabla 10. Disponibilidad estimada de tractores en diferentes países del área mediterránea

\begin{tabular}{|l|c|}
\hline \multicolumn{1}{|c|}{ País } & Tractores en servicio / 1000 ha de superficie cultivada \\
\hline Argelia & 23,3 \\
Marruecos & s.d. \\
Túnez & 11,2 \\
España & 81,2 \\
Israel & 79,3 \\
Italia & 245,7 \\
\hline
\end{tabular}

Estimación a partir de datos de FAO del año 2008, excepto Italia $(2002)^{21}$

Los últimos datos estadísticos de FAO sobre este concepto para Marruecos son de 1999, con un valor de 43.226 unidades (que equivaldrían aproximadamente a 4,7 tractores por cada 1000 ha). Aparte del dato correspondiente a Italia, que puede resultar desmesurado, se observa que, con independencia de que haya variaciones debidas a diferencias en los sistemas de explotación y en las especies cultivadas, hay una notable diferencia entre los países del Magreb y los restantes, lo que indica una menor mecanización en los primeros. Es cierto que, en ocasiones, los sistemas de manejo de ciertas especies son mucho más intensivos en el uso de la mano de obra, y que esta tendencia puede venir promovida por factores como dimensión de parcelas, dificultades orográficas, etc., pero queda como referencia el menor uso de medios técnicos avanzados. El trabajo de Soler y Arroyo también hace algunas consideraciones sobre el uso de productos fitosanitarios, que respaldan esta tesis de que aún es posible avanzar en la tecnificación del sector agrario de estos países y, consecuentemente, en su productividad.

El potencial productivo puede reforzarse, como ya se ha indicado, en la medida en que se pueda aprovechar mejor el agua disponible. En este sentido, y resumiendo algunas ideas de otras aportaciones $\left(Z_{a r e b}{ }^{22}\right.$ ) y de diversas fuentes, se pueden apuntar algunas de las iniciativas que se contemplan en el escenario actual.

En Argelia, el Plan Quinquenal 2010-2014 contempla, en el marco de la Política de Renovación Agrícola y Rural (PRAR), diferentes acciones en materia de aumento de los recursos de agua. Hay planes para la transferencia de agua a zonas del Sur y al área de los Hauts Plateaux, con seis grandes proyectos de trasvase; para la construcción de 19 nuevas presas y la reconstrucción de cuatro embalses ya existentes (pasando a 9,1 $\mathrm{km}^{3}$ de capacidad embalsada en 2014); la implementación de una amplia red de desalación de agua de mar, y la puesta en marcha de 40 estaciones de depuración de aguas residuales, así como otras medidas.

\footnotetext{
${ }^{21}$ FAO (2012): Base de datos FAOSTAT, Estadísticas de maquinaria, en http://faostat.fao.org/site/576/default.aspx \#ancor.

${ }^{22}$ Zareb, Djamel: "Les programmes de développement en eau dans la stratégie de développement de la securité alimentaire en Algerie", Presentation, Facultad de CC. Políticas y Sociología, Madrid (30 de Octubre, 2012).
} 
Si bien estos planes tienen como objetivo primario la mejora del suministro de agua para la población, la mayor disponibilidad de agua y la posibilidad de reutilización agrícola de aguas procedentes de otros usos pueden ayudar a mejorar la disponibilidad de agua para el riego.

En cuanto a Marruecos, la Nueva Estrategia del Agua planteada en 2009 considera, en un escenario de acción entre 2010 y 2030, una serie de actuaciones. Por vía de la demanda de agua, se contempla un programa de conversión masiva a sistemas de riego localizado, mucho más eficientes y ahorradores de agua; además, la mejora de las redes urbanas de distribución y de las redes de abastecimiento a los regadíos. Por vía de la oferta, se planea la construcción de 60 grandes presas y del orden de un millar de presas pequeñas; la realización de trasvases Norte-Sur, y la implementación de sistemas de desalación y recuperación y reutilización de aguas residuales.

En Túnez, ya se ha apuntado (Tabla 6) que la superficie total regable es cercana a las 400.000 ha. Estas zonas regables -que representan menos del $8 \%$ de la superficie agrícola útil- contribuyen de manera importante a la actividad socioeconómica del país. En efecto, la producción agrícola del regadío representa del 30 al $35 \%$ de la producción agrícola total y del 13 al $15 \%$ del PIB. Desde 1995 se han venido realizando acciones de modernización de zonas regables en el marco del Programa Nacional de Ahorro de Agua de Riego. La modernización de los regadíos se ha traducido en un aumento de la implantación de los sistemas de riego modernos (localizado, aspersión y riego por gravedad mejorado). Estas mejoras se han realizado ya sobre más de las tres cuartas partes de la superficie regada.

\section{Conclusiones}

En síntesis, desde el punto de vista de las restricciones y posibilidades del potencial productivo agrario en el Magreb Central se pueden considerar los aspectos tecnológicos y agronómicos que se exponen en los párrafos siguientes.

La producción agraria en la zona está sujeta a limitaciones severas en lo relativo a la superficie utilizable. Esas limitaciones no son de escasez de territorio, sino que se refieren tanto a la disponibilidad de tierra apta para el cultivo como a sus posibilidades de fertilidad y manejo. Las limitaciones de superficie están asociadas también a condicionantes climáticos, que inhabilitan para el cultivo o la explotación ganadera amplias zonas. Un elemento asociado a considerar es el riesgo de deterioro de la calidad del suelo, que en su máximo grado conduce a la desertificación.

En las zonas útiles para la agricultura, el clima mediterráneo a árido supone una variabilidad interanual notable de las condiciones meteorológicas, lo que genera oscilaciones importantes de la producción entre campañas.

A pesar de lo anterior, existe potencial de crecimiento, en la medida en que se intensifique y tecnifique el uso de algunos factores de producción (nutrientes, mecanización, fitosanitarios, riego,...). Ese potencial ya ha sido empleado y se está poniendo en juego en los diferentes países del área; las posibilidades de incremento son, por tanto, variables. 
Es muy conveniente un esfuerzo en el desarrollo e implantación de estrategias adecuadas de manejo de cultivos en zonas áridas. Ya existen metodologías para ello, que habría que adaptar (en su caso) y difundir.

La mejora de la eficiencia de los sistemas de riego puede ser un factor de primer orden, sobre el que ya se viene trabajando en los tres países. También es importante el incremento racional de la mecanización, así como el aumento de uso de otros factores empleados en la producción moderna, especialmente productos fitosanitarios y programas de sanidad animal.

La cualificación profesional a todos los niveles, a la que se alude también como factor a promover en la aportación de Djamel Zareb ${ }^{23}$, es imprescindible para que el resto de medidas y mejoras alcancen sus mejores resultados. 